J. DIFFERENTIAL GEOMETRY

96 (2014) 303-351

\title{
ERGODICITY AND INTERSECTIONS OF NODAL SETS AND GEODESICS ON REAL ANALYTIC SURFACES
}

\author{
Steve ZeLDitCH
}

\begin{abstract}
We consider the intersections of the complex nodal set $\mathcal{N}_{\lambda_{j}}^{\mathbb{C}}$ of the analytic continuation of an eigenfunction of $\Delta$ on a real analytic surface $\left(M^{2}, g\right)$ with the complexification of a geodesic $\gamma$. We prove that if the geodesic flow is ergodic and if $\gamma$ is periodic and satisfies a generic asymmetry condition, then the intersection points $\mathcal{N}_{\lambda_{j}}^{\mathbb{C}} \cap \gamma_{x, \xi}^{\mathbb{C}}$ condense along the real geodesic and become uniformly distributed with respect to its arc-length. We prove an analogous result for non-periodic geodesics except that the 'origin' $\gamma_{x, \xi}(0)$ is allowed to move with $\lambda_{j}$.
\end{abstract}

\section{Contents}

0.1. A key Lemma 306

0.2. Statement of results for asymmetric periodic geodesics on surfaces $\quad 308$

0.3. Statement of results for non-periodic geodesics 308

0.4. Discussion of the proofs 310

0.5. Generalizations 313

0.6. Acknowledgements 314

1. Geometry of geodesics and Grauert tubes 314

1.1. Geodesic flow in the real domain 314

1.2. Grauert tubes 314

1.3. Complexified Hamiltonian flow 316

1.4. Hamiltonian flow of $\sqrt{\rho}$ and complexified geodesics 317

1.5. Kähler isometric embedding of geodesics 318

2. Szegö kernels on boundaries of Grauert tubes 319

2.1. Szegö projector on boundaries of Grauert tubes $\quad 319$

3. Poisson kernels and their analytic continuations 321

3.1. Poisson kernel and Szegö kernel 324

Research partially supported by NSF grants \# DMS-0904252 and DMS-1206527. Received 11/30/2012. 
3.2. Pointwise Weyl laws and norms for complexifed eigenfunctions

4. The wave group in the complex domain 326

4.1. The wave group $\tilde{V}_{\tau}^{t} \quad 326$

4.2. $V_{\tau}^{t}$ as a Fourier integral Toeplitz operator $\quad 327$

4.3. $V_{\tau}^{t}$ as a Toeplitz Fourier integral operator 328

5. Restriction to geodesics I: Intertwining relations 329

5.1. Proof of Lemma 5.3 331

6. Lebesgue limits of matrix elements: Proof of Proposition 2333

6.1. Lebesgue limits for the family of translates 336

7. Growth rates of restricted eigenfunctions: Proof of Lemma 1337

7.1. Hartogs theorem for the family of translates 339

8. Periodic geodesics on surfaces: Proof of Proposition $1 \quad 340$

8.1. Mass in the highest allowed modes 341

8.2. Completion of proof of Proposition 1 for periodic geodesics 342

9. Non-periodic geodesics: Proof of Theorem 2

9.1. Analytic convergence factors 342

9.2. Logarithmic asymptotics 345

9.3. Completion of proof of Theorem 2

10. Appendix 346

10.1. Quantum ergodic restriction in the real domain 346

10.2. Fourier integral distributions with complex phase 348

10.3. Complex canonical relation of $\Pi_{\tau} \quad 349$

References $\quad 350$

This article is concerned with the 'complex geometry' of nodal sets of Laplace eigenfunctions on real analytic Riemannian surfaces $\left(M^{2}, g\right)$ with ergodic geodesic flow. All but one of the methods are valid in all dimensions, so until it is necessary to specialize to surfaces we consider Riemannian manifolds $\left(M^{m}, g\right)$ of general dimension $m$. Let $\left\{\varphi_{j}\right\}$ be an orthonormal basis of eigenfunctions of the Laplacian $\Delta$ of $(M, g)$,

$$
\Delta \varphi_{j}=\lambda_{j}^{2} \varphi_{j}, \quad\left\langle\varphi_{j}, \varphi_{k}\right\rangle=\delta_{j k},
$$

where $\lambda_{0}=0<\lambda_{1} \leq \lambda_{2} \leq \cdots$ and where $\langle u, v\rangle=\int_{M} u v d V_{g}\left(d V_{g}\right.$ being the volume form). When the geodesic flow $G^{t}: S_{g}^{*} M \rightarrow S_{g}^{*} M$ is ergodic on the unit (co-)tangent bundle, the 'random wave model' for eigenfunctions predicts that the nodal sets

$$
\mathcal{N}_{\varphi_{j}}=\left\{x \in M: \varphi_{j}(x)=0\right\}
$$


become equidistributed with respect to the volume form in the sense that

(1)

$$
\frac{1}{\mathcal{H}^{m-1}\left(\mathcal{N}_{\varphi_{j}}\right)} \int_{\mathcal{N}_{\varphi_{j}}} f d \mathcal{H}^{m-1} \rightarrow \frac{1}{\operatorname{Vol}(M, g)} \int_{M} f d V_{g}, \quad(\forall f \in C(M)) .
$$

Here, $\mathcal{H}^{m-1}\left(\mathcal{N}_{\varphi_{j}}\right)$ denotes the hypersurface volume; we refer to $[\mathbf{Z 4}]$ for background. This prediction appears to lie far beyond the scope of current mathematical techniques. But we aim to show that something quite close to (1) can be proved for intersections of complex nodal lines and complexified geodesics on real analytic surfaces with ergodic geodesic flow. Roughly speaking, we show that in the complex domain, as $\lambda \rightarrow \infty$, the intersections of nodal sets with generic periodic and non-periodic geodesics condense along the underlying real geodesic and become uniformly distributed relative to its arclength. Much of the proof generalizes to any real analytic curve on a surface or to a real analytic hypersurface in higher dimensions, but the case of geodesics seems to us special and interesting enough to deserve a separate treatment; the potential generalizations are discussed at the end of the introduction.

To state our results, we introduce some notation. We recall that any real analytic manifold $M$ admits a Bruhat-Whitney complexification $M_{\mathbb{C}}[\mathbf{B W}]$, and that for any real analytic metric $g$ all of the eigenfunctions $\varphi_{j}$ extend holomorphically to a fixed open neighborhood $M_{\epsilon}$ of $M$ in $M_{\mathbb{C}}$ called a Grauert tube. We denote the holomorphic extension by $\varphi_{j}^{\mathbb{C}}$. The 'radius' (in the imaginary direction) of an open neighborhood is measured by the Grauert tube function $\sqrt{\rho}$ and we denote the level set $\sqrt{\rho}=\tau$ by $\partial M_{\tau}$ for $\tau \leq \epsilon$ (see $\S 1$ for background). The complex nodal sets are defined by

$$
\mathcal{N}_{\varphi_{j}^{\mathbb{C}}}=\left\{\zeta \in M_{\epsilon}: \varphi_{j}^{\mathbb{C}}(\zeta)=0\right\} .
$$

We propose to intersect these nodal sets with the (image of the) complexification of an arc-length parameterized geodesic

$$
\gamma_{x, \xi}: \mathbb{R} \rightarrow M, \quad \gamma_{x, \xi}(0)=x, \quad \gamma_{x, \xi}^{\prime}(0)=\xi \in T_{x} M .
$$

As recalled in $\S 1, \gamma_{x, \xi}$ admits an analytic continuation

$$
\gamma_{x, \xi}^{\mathbb{C}}: S_{\epsilon} \rightarrow M_{\epsilon}
$$

to the strip

$$
S_{\epsilon}=\{(t+i \tau \in \mathbb{C}:|\tau| \leq \epsilon\} .
$$

When we freeze $\tau$ we simplify the notation to

$$
\gamma_{x, \xi}^{\tau}(t):=\gamma_{x, \xi}^{\mathbb{C}}(t+i \tau) .
$$


The intersection points of $\gamma_{x, \xi}^{\mathbb{C}}$ and $\mathcal{N}_{\varphi_{j}}^{\mathbb{C}}$ correspond to the zeros of the pullback $\left(\gamma_{x, \xi}^{\mathbb{C}}\right)^{*} \varphi_{j}^{\mathbb{C}}$. We encode this discrete set by the measure

$$
\left[\mathcal{N}_{\lambda_{j}}^{\gamma_{x, \xi}^{\mathbb{C}}}\right]=\sum_{(t+i \tau): \varphi_{j}^{\mathbb{C}}\left(\gamma_{x, \xi}^{\mathbb{C}}(t+i \tau)\right)=0} \delta_{t+i \tau}
$$

Definition 1. Let $\mathcal{S}=\left\{j_{k}\right\} \subset \mathbb{N}$ be a subsequence of the positive integers. We say that the intersection points of the complex nodal sets $\mathcal{N}_{\lambda_{j_{k}}}^{\mathbb{C}}$ and the complexified geodesic $\gamma_{x, \xi}^{\mathbb{C}}$ for the subsequence $\mathcal{S}$ condense on the real geodesic and become uniformly distributed with respect to arc-length if, for any $f \in C_{c}\left(S_{\epsilon}\right)$,

$$
\lim _{k \rightarrow \infty} \frac{1}{\lambda_{j_{k}}} \sum_{(t+i \tau): \varphi_{j_{k}}^{\mathbb{C}}\left(\gamma_{x, \xi}^{\mathbb{C}}(t+i \tau)\right)=0} f(t+i \tau)=\frac{1}{\pi} \int_{\mathbb{R}} f(t) d t .
$$

That is, $\frac{1}{\lambda_{j_{k}}}\left[\mathcal{N}_{\lambda_{j}}^{\gamma_{x, \xi}^{\mathbb{C}}}\right] \rightarrow \frac{1}{\pi} \delta_{0}(\tau) d t d \tau$ in the sense of measures.

The first result of this article (Theorem 1) gives a sufficient condition on a periodic geodesic $\gamma_{x, \xi}$ for the existence of a subsequence $\mathcal{S}_{x, \xi}$ of density one of the $\left\{\lambda_{j}\right\}$ for which $\left\{\mathcal{N}_{\lambda_{j_{k}}}\right\}$ satisfies the condition of Definition 1. The condition is that the QER (quantum ergodic restriction) result of $[\mathbf{T Z}]$ is valid for the geodesic. As recalled in $\S 10.1$ (see especially Theorem 10.2) the QER result states that restrictions of eigenfuntions $\left.\varphi_{j_{k}}\right|_{\gamma_{x, \xi}}$ to a (real) geodesic are quantum ergodic along $\gamma_{x, \xi}$ as long as $\gamma_{x, \xi}$ is asymmetric (as a hypersurface of $M$ ) with respect to the geodesic flow. The asymmetry condition (Definition 4) pertains to pairs of geodesics $\alpha(t), \alpha^{*}(t): \mathbb{R} \rightarrow M$, with $\alpha(0)=\alpha^{*}(0) \in \gamma_{x, \xi}$ (i.e. the trace of $\left.\gamma_{x, \xi}\right)$ and with $\alpha^{*^{\prime}}(0)$ equal to the reflection through $T_{\alpha(0)} \gamma_{x, \xi}$ of $\alpha^{\prime}(0)$. The condition is that $\alpha(t)$ and $\alpha^{*}(t)$ almost never return to $\gamma_{x, \xi}$ at the same time and the same point. This is a generic condition on a closed geodesic and also holds for any Birkhoff regular geodesic. Since geodesics are hypersurfaces only when $\operatorname{dim} M=2$, the result on intersections of periodic geodesics and nodal sets is only proved in that dimension. Results independent of QER hold in all dimensions.

The second result ( Theorem 2) is an analogous result for non-periodic geodesics, such as Birkhoff regular ones. The result is somewhat weaker due to the non-compactness of non-periodic geodesics and the resulting problems with escape of mass at parameter time infinity.

0.1. A key Lemma. Before stating the Theorems precisely, we state a key Lemma which is valid in all dimensions and which reduces the equidistribution of zeros in the ergodic case to growth estimates. It is ultimately based on the Proposition 2 which we state after some further preliminaries. 
The starting point is the Poincaré-Lelong formula, according to which we may express the current of summation over the intersection points in (6) in the form,

$$
\left[\mathcal{N}_{\lambda_{j}}^{x, \xi}\right]=\frac{i}{\pi} \partial \bar{\partial}_{t+i \tau} \log \left|\gamma_{x, \xi}^{*} \varphi_{\lambda_{j}}^{\mathbb{C}}(t+i \tau)\right|^{2} .
$$

This formula holds for the complexification of any real analytic curve. It follows from (7) that the main step in the proofs of all the theorems is to obtain the asymptotics of the sequence

$$
v_{j}^{x, \xi}:=\frac{1}{\lambda_{j}} \log \left|\gamma_{x, \xi}^{*} \varphi_{\lambda_{j}}^{\mathbb{C}}(t+i \tau)\right|^{2}, \quad\left((x, \xi) \in S^{*} M\right)
$$

of subharmonic functions on a strip $S_{\epsilon} \subset \mathbb{C}$.

Next we use the following compactness result, which combines a standard compactness Lemma due to Hartogs, H. Cartan and L. Hörmander with a stronger conclusion that is ultimately based on Proposition 2 below. We use the notation $v^{*}$ for the USC (upper semi-continuous) regularization of $v$. For background we refer to [Ho2] (Theorems 3.2.123.2.13).

Lemma 1. For any compact analytic Riemannian manifold $(M, g)$, and any $(x, \xi) \in S^{*} M$, the family of subharmonic functions

$$
\mathcal{F}^{x, \xi}:=\left\{v_{j}^{x, \xi}(t+i \tau), j=1,2, \ldots\right\}
$$

on the strip $S_{\epsilon}$ is precompact in $L_{l o c}^{1}\left(S_{\epsilon}\right)$ as long as it does not converge uniformly to $-\infty$ on all compact subsets of $S_{\epsilon}$. Moreover:

- For all $(x, \xi), \lim \sup _{k \rightarrow \infty} v_{k}^{x, \xi}(t+i \tau) \leq 2|\tau|$.

- Let $\left\{v_{j_{k}}^{x, \xi}\right\}$ be any subsequence of $\left\{v_{j}^{x, \xi}\right\}$ with a unique $L_{l o c}^{1}$ limit $v$ on $S_{\epsilon}$ and let $v^{*}$ be its USC regularization. Then if $v^{*}<2|\tau|-\epsilon$ on an open set $U \subset S_{\epsilon}$ then $v^{*} \leq 2|\tau|-\epsilon$ for $\tilde{U}=\bigcup_{t \in \mathbb{R}}(U+t)$ and

$$
\limsup _{k \rightarrow \infty} v_{j_{k}} \leq 2|\tau|-\epsilon \text { on } \tilde{U} .
$$

The upper bounds follow from the global upper bound

$$
\limsup _{k \rightarrow \infty} \frac{1}{\lambda_{j}} \log \left|\varphi_{j_{k}}(\zeta)\right|^{2} \leq 2 \sqrt{\rho}(\zeta)
$$

everywhere on $\partial M_{\tau}$ proved in $[\mathbf{Z}]$; we review it in $\S 3.2$. However, it is not generally true that if a limit $v^{*}$ is $<2|\tau|-\epsilon$ on some open set then it is globally $<2|\tau|-\epsilon$ on $\mathbb{R}$. This is where Proposition 2 is used.

Since there is no unique choice of origin along (the trace of) the parameterized geodesic $\gamma_{x, \xi}$ it is natural to consider the enlarged family

$$
\mathcal{F}_{\mathbb{R}}^{x, \xi}:=\bigcup_{s \in \mathbb{R}} \mathcal{F}^{G^{s}(x, \xi)}
$$


of translates of $\gamma_{x, \xi}^{*} \varphi_{j}$ for $j=1,2, \ldots$ The compactness result of Lemma 1 generalizes to this family; we refer to Lemma 7.1 for the statement we need.

0.2. Statement of results for asymmetric periodic geodesics on surfaces. The first result pertains to periodic geodesics on surfaces which satisfy the asymmetry condition (Definition 4). The asymmetry condition is needed to rule out obvious counter-examples such as when $\gamma_{x, \xi}$ is the fixed point set of an isometric involution; then "odd" eigenfunctions under the involution will vanish everywhere on the geodesic. As mentioned above, the asymmetry condition arose in [TZ] (see also $[\mathbf{D Z}]$ ) as the condition that $\gamma_{x, \xi}$ have the QER (quantum ergodic restriction) property, i.e that there exists a full density set of eigenfunctions $\left\{\varphi_{j_{k}}\right\}$ which are quantum ergodic when restricted to $\gamma_{x, \xi}$ (see Theorems 10.1 -10.2). The generic asymmetry of periodic geodesics on hyperbolic surfaces is proved in [TZ] and the discussion is almost the same for any surface of negative curvature. Hence we do not discuss existence of asymmetric periodic geodesics in this article.

Theorem 1. Let $\left(M^{2}, g\right)$ be a real analytic Riemannian surface with ergodic geodesic flow. Let $\gamma_{x, \xi}$ be a periodic geodesic satisfying the assymetry QER hypothesis of Definition 4. Then there exists a subsequence of eigenvalues $\lambda_{j_{k}}$ of density one such that the equi-distribution result in Definition 1 holds.

The main Proposition is:

Proposition 1. (Growth saturation) If $\gamma_{x, \xi}$ is a periodic geodesic which satisfies the QER asymmetry condition (Definition 4) along compact arcs, then there exists a subsequence $\mathcal{S}_{x, \xi}$ of density one so that, for all $\tau<\epsilon$,

$$
\lim _{k \rightarrow \infty} \frac{1}{\lambda_{j_{k}}} \log \left|\gamma_{x, \xi}^{\tau *} \varphi_{\lambda_{j_{k}}}^{\mathbb{C}}(t+i \tau)\right|^{2}=2|\tau| \quad \text { in } L_{l o c}^{1}\left(S_{\tau}\right) .
$$

The subsequence $\mathcal{S}_{x, \xi}$ is the ergodic sequence along $\gamma_{x, \xi}$ given by Theorem 10.1 .

Proposition 1 immediately implies Theorem 1 since we can apply $\partial \bar{\partial}$ to the $L^{1}$ convergent sequence $\frac{1}{\lambda_{j_{k}}} \log \left|\gamma_{x, \xi}^{*} \varphi_{\lambda_{j_{k}}}^{\mathbb{C}}(t+i \tau)\right|^{2}$ to obtain a weakly convergence sequence of measures tending to $2 \partial \bar{\partial}|\tau|$.

Proposition 1 has an analogue for any real analytic curve but the exact formula is special to geodesics and arises because complex geodesics are isometric embeddings to Grauert tubes (see §1.5). In general, the growth rates of restrictions depend on the curve.

0.3. Statement of results for non-periodic geodesics. We next consider non-periodic geodesics. The non-compactness of $\mathbb{R}$ may allow 
the $L^{2}$ mass of the restricted eigenfunctions (in the real or complex domain) to escape 'to infinity' along the parameter interval $\mathbb{R}$ of the geodesic as $\lambda_{j} \rightarrow \infty$. That is, $\left|\varphi_{j}^{\mathbb{C}}\right|_{\gamma_{x, \xi}^{\tau}}$ might achieve growth saturation only on intervals $I_{j} \subset \mathbb{R}$ which get translated to infinity as $j \rightarrow \infty$. Viewed on the compact manifold $M$, the 'high mass' intervals might move along $\gamma_{x, \xi}$, and in the limit the saturating mass might jump to another geodesic.

To gain some partial compactness, we consider the two-parameter family (11) of restrictions $\gamma_{G^{s}(x, \xi)}^{*} \varphi_{j}$ as $s, \lambda_{j}$ vary. For fixed $\lambda_{j}$ this is the family of translates of $\gamma_{x, \xi}^{*} \varphi_{j}$. Of course, this family is non-compact in $C_{b}(\mathbb{R})$ since $\gamma_{x, \xi}^{*} \varphi_{j}$ is not an almost-periodic function.

Theorem 2. Let $\left(M^{2}, g\right)$ be a real analytic Riemannian surface with ergodic geodesic flow. Let $\gamma_{x, \xi}$ be a non-periodic geodesic satisfying the assymetry QER hypothesis of Definition 4. Then there exists a subsequence of eigenvalues $\lambda_{j_{k}}$ of density one and a sequence $\left\{N_{k}\right\} \subset \mathbb{R}$ such that for any $f \in C_{c}\left(S_{\epsilon}\right)$,

$$
\lim _{k \rightarrow \infty} \sum_{(t+i \tau): \gamma_{(x, \xi)}^{*}} \sum_{\varphi_{j_{k}}^{\mathbb{C}}\left(t+N_{k}+i \tau\right)=0} f(t+i \tau)=\frac{1}{\pi} \int_{\mathbb{R}} f(t) d t .
$$

Thus, we obtain a result parallel to that of Theorem 1 except that we may have to translate the origin $x$ of $\gamma_{x, \xi}$ unbounded parameter distances along the geodesic.

These concentration- equidistribution results are 'restricted' versions of the result of $[\mathbf{Z}]$, which states that for real analytic $(M, g)$ with ergodic geodesic flow,

$$
\left\langle\psi,\left[\frac{1}{\lambda_{j}} \mathcal{N}_{\varphi_{\lambda_{j}}^{\mathbb{C}}}\right]\right\rangle \rightarrow \frac{1}{\pi} \int_{M_{\tau}} \psi \omega^{m-1} d d^{c} \sqrt{\rho}
$$

for a density one subsequence of ergodic eigenfunctions. Here, $\omega=d d^{c} \rho$ is the Kähler metric on the Grauert tube induced by $g$ (see $[\mathbf{G S 1}, \mathbf{L S}$ ] and $\S 1$ ). An important point to observe is that $d d^{c} \sqrt{\rho}$ is singular along the real domain, indicating that complex zeros concentrate along the totally real submanifold $M$. Our results show that the singularity is magnified under restriction to asymmetric geodesics, indeed it becomes a delta function along the real geodesic.

It follows that there exist many "nearly real" intersections of a complex geodesic with the complex nodal line when the geodesic flow is ergodic (i.e. zeros of $\gamma_{x, \xi}^{*} \varphi_{j}^{\mathbb{C}}$ whose imaginary parts tend to zero with $\left.\lambda_{j}\right)$. It would be very interesting to know the proportion of "truly real" intersection points among these nearly real ones. There are very few lower bounds known at present on the number of real intersection 
points, except in special cases such as separation of variables eigenfunctions or eigenfunctions on flat tori $[\mathbf{B R}]$ or special eigenfunctions and the geodesics on the modular hyperbolic surface [GRS].

0.4. Discussion of the proofs. The proofs involve several principles which played no role in the global result (12). Some hold in much greater generality and some are specific to geodesics. At the end of the introduction we discuss the potential generalizations.

One of the main ingredients in the proof is an invariance principle for restrictions to geodesics in the complex domain that is a simple kind of QUER (quantum uniquely ergodic restriction) principle. The main statement (Lemma 2) proves the translation invariance of the limit measures of $L^{2}$ normalizations of $\left|\gamma_{x, \xi}^{*} \varphi_{j}^{\mathbb{C}}(t+i \tau)\right|^{2}$ along intervals in each horizontal line of $S_{\tau}$. Intuitively, it is the restricted version of the standard fact that Wigner measures of eigenfunctions are almost invariant under the geodesic flow. Since we are restricting to a single geodesic, the result should be translation invariance of the limit measures. But we obviously need to normalize $\gamma_{x, \xi}^{*} \varphi_{j}^{\mathbb{C}}(t+i \tau)$ along horizontal lines to obtain a bounded family of measures. The non-compactness of $\mathbb{R}$ in the case of non-periodic geodesics forces us to work on compact sub-intervals.

In the case of periodic geodesics, we can normalize $\gamma_{x, \xi}^{*} \varphi_{j}^{\mathbb{C}}(t+i \tau)$ for each $\tau$ by dividing by its $L^{2}$ norm on the horizontal interval $t \in[0, L]$ where $L$ is the period of $\gamma_{x, \xi}$ in the real domain (hence also in the complex domain). When $\gamma_{x, \xi}$ is a non-periodic geodesic, there is no canonical choice of normalization and therefore we consider all possibly choices. When we pull back under $\gamma_{x, \xi}^{\tau}$, we consider all possible renormalizations along intervals $I$ as follows:

Definition 2. Let $I \subset \mathbb{R}$ be an interval and let $\mathbf{I}_{\tau} \subset \partial S_{\tau}=\{t+i \tau$ : $t \in I\}$ be the indicated segment of $\partial S_{\tau}$. Then define

$$
U_{j}^{\mathbf{I}_{\tau}, x, \xi}:=\frac{\left.\gamma_{x, \xi}^{\tau *} \varphi_{j}^{\mathbb{C}}\right|_{\partial S_{\tau}}}{\left\|\left.\gamma_{x, \xi}^{\tau *} \varphi_{j}^{\mathbb{C}}\right|_{\partial S_{\tau}}\right\|_{L^{2}\left(I_{\tau}, d t\right)}}, \quad\left(\text { i.e. } \quad \int_{\mathbf{I}_{\tau}}\left|U_{j}^{\mathbf{I}_{\tau}, x, \xi}\right|^{2} d t=1\right),
$$

where

$$
\|f\|_{L^{2}\left(\mathbf{I}_{\tau}\right)}=\int_{t \in \mathbf{I}_{\tau}}\left|f\left(G^{t+i \tau}(x, \xi)\right)\right|^{2} d t .
$$

We then associate Wigner measures to normalized complexified eigenfunctions. As will be explained below, in the complex domain the relevant theory of pseudo-differential operators is the Toeplitz calculus of $[\mathbf{B o G u}]$. This reflects the fact that restricted complexified eigenfuntions concentrate microlocally on the tangent directions to $\gamma_{x, \xi}$. Hence Wigner measures are defined simply by multiplication operators along $\partial S_{\tau}$ :

Definition 3. Let $a \in C_{c}^{\infty}\left(\partial S_{\tau}\right)$ and set 


$$
\int_{\partial S_{\tau}} a(t) d W_{j}^{\mathbf{I}_{\tau}, x, \xi}(t):=\left\langle a U_{j}^{\mathbf{I}_{\tau}, x, \xi}, U_{j}^{\mathbf{I}_{\tau}, x, \xi}\right\rangle=\int_{\partial S_{\tau}} a\left|U_{j}^{\mathbf{I}_{\tau}, x, \xi}\right|^{2} d t .
$$

Our aim is then to determine the weak* limits of

$$
\left|U_{j}^{\mathbf{I}_{\tau}, x, \xi}\right|^{2} \text { on } \mathbf{I}_{\tau} \text { and on general compact sets of } \partial S_{\tau} \text {. }
$$

Since $\left|U_{j}^{\mathbf{I}_{\tau}, x, \xi}\right|^{2}$ is normalized to have mass one on $\mathbf{I}_{\tau}$ it forms a precompact family of probability measures on $\mathbf{I}_{\tau}$. The following Proposition asserts that the Wigner distributions are asymptotically invariant under translation.

Proposition 2. (Lebesgue limits) Let $(M, g)$ be a real analytic Riemannian manifold of any dimension $m$, Let $(x, \xi) \in S^{*} M$ be any point and let $\mathbf{I}_{\tau}$ be the interval defined above. Then as long as $\gamma_{x, \xi}^{*} \varphi_{j} \neq 0$ (identically), the sequence $\left\{\left|U_{j}^{\mathbf{I}_{\tau}, x, \xi}\right|^{2}\right\}$ is QUE on $\mathbf{I}_{\tau}$ with limit measure given by normalized Lebesgue measure on $\mathbf{I}_{\tau}$. That is, for any $a \in C_{c}^{\infty}\left(\mathbf{I}_{\tau}\right)$, we have

$$
\lim _{j \rightarrow \infty} \int_{\mathbf{I}_{\tau}} a(t) d W_{j}^{\mathbf{I}_{\tau}, x, \xi}=\frac{1}{\left|\mathbf{I}_{\tau}\right|} \int_{\mathbf{I}_{\tau}} a(s) d s .
$$

The proof of Proposition 2 uses the Toeplitz Fourier integral operator calculus of Boutet de Monvel-Guillemin [BoGu]. Toeplitz operators arise in the complex domain because the restriction $\gamma_{x, \xi}^{\tau *} V_{\tau}^{t}$ of the wave group $V_{\tau}^{t}$ on $\partial M_{\tau}$ is a Toeplitz Fourier integral operator (we refer to $\S 4$ for the notation). This is the analogue in the complex domain of the operator $W=\gamma_{x, \xi}^{*} U(t)$ studied in [ $\left.\mathbf{T Z}\right]$. In the real domain this operator is a Fourier integral operator with a one-sided fold singularity; in the complex domain the analogous operator is of a very different type: it is a Toeplitz Fourier integral which microlocally lives on the tangent line to the geodesic. The main point of the proof is to show that the Wigner distributions $U_{j}^{\mathbf{I}_{\tau}, x, \xi}$ are almost invariant under time translation. But the only translation invariant measures on $\mathbb{R}$ are constant multiples of Lebesgue measure. Since we normalized the Wigner distributions to have integral 1 over $\mathbf{I}_{\tau}$, the constant must be one on that interval.

The behavior of the local mass on general intervals is not clear apriori when $\gamma_{x, \xi}$ is non-periodic, especially at parameter distances $t$ exceeding the 'Eherenfest time' $\log \lambda_{j}$, where the remainders in Egorov type theorems break down. The weak $*$ limits cannot be deduced from those on $\partial M_{\epsilon}$ (which were studied in $[\mathbf{Z}]$ ) since weak* convergence is not preserved by restriction to sets of measure zero.

Proposition 2 combines with Lemma 1 as follows: If any limit is $<\tau-\epsilon$ on an open set, then by Proposition 2 it has to be $<\tau-\epsilon$ on all of $\partial S_{\tau}$. Otherwise, the normalizations of $U_{j}^{\mathbf{I}_{\tau}, x, \xi}$ would have different 
exponential orders and Proposition 2 could not be true for every interval $I$. We rely on the fact that Proposition 2 holds simultaneously for all of the normalized pullbacks in Definition 2.

We now sketch the proof of Theorem 1 on periodic geodesics and of Proposition 1 to highlight the differences between restriction to geodesics in the real and complex domains and to indicate the kinds of new phenomena that are needed in the proof. To prove Proposition 1 in the case of periodic $\gamma_{x, \xi}$, we first prove an integrated version for $L^{2}$ norms.

Lemma 2. Let $\gamma_{x, \xi}$ be a periodic geodesic of period L. Assume that $\left\{\varphi_{j}\right\}$ satisifies QER along the periodic geodesic $\gamma_{x, \xi}$. Let $\left\|\gamma_{x, \xi}^{\tau *} \varphi_{j}^{\mathbb{C}}\right\|_{L^{2}\left(\partial S_{\tau}\right)}^{2}$ be the $L^{2}$-norm of the complexified restriction of $\varphi_{j}$ to a period cell $\partial S_{\tau}^{L}$ of $\partial S_{\tau}$. Then,

$$
\lim _{\lambda_{j} \rightarrow \infty} \frac{1}{\lambda_{j}} \log \left\|\gamma_{x, \xi}^{\tau *} \varphi_{j}^{\mathbb{C}}\right\|_{L^{2}\left(\partial S_{\tau}^{L}\right)}^{2}=2|\tau| .
$$

To prove Lemma 2, we study the orbital Fourier series of $\gamma_{x, \xi}^{\tau *} \varphi_{j}$ and of its complexification. The orbital Fourier coefficients are

$$
\nu_{\lambda_{j}}^{x, \xi}(n)=\frac{1}{L} \int_{0}^{L} \varphi_{\lambda_{j}}\left(\gamma_{x, \xi}(t)\right) e^{-\frac{2 \pi i n t}{L}} d t,
$$

and the orbital Fourier series is

$$
\varphi_{\lambda_{j}}\left(\gamma_{x, \xi}(t)\right)=\sum_{n \in \mathbb{Z}} \nu_{\lambda_{j}}^{x, \xi}(n) e^{\frac{2 \pi i n t}{L}} .
$$

Hence the analytic continuation of $\gamma_{x, \xi}^{*} \varphi_{j}$ is given by

$$
\varphi_{\lambda_{j}}^{\mathbb{C}}\left(\gamma_{x, \xi}(t+i \tau)\right)=\sum_{n \in \mathbb{Z}} \nu_{\lambda_{j}}^{x, \xi}(n) e^{\frac{2 \pi i n(t+i \tau)}{L}} .
$$

By the Paley-Wiener theorem for Fourier series, the series converges absolutely and uniformly for $|\tau| \leq \epsilon_{0}$. The growth rate of $\varphi_{\lambda_{j}}^{\mathbb{C}}\left(\gamma_{x, \xi}(t+i \tau)\right)$ is thus intimately related to the joint asymptotics of the Fourier coefficients $\nu_{\lambda_{j}}^{x, \xi}(n)$ in $\left(\lambda_{j}, n\right)$. We use the QER hypothesis in the following way:

Lemma 3. Suppose that $\left\{\varphi_{\lambda_{j}}\right\}$ is QER along the periodic geodesic $\gamma_{x, \xi}$. Then for all $\epsilon>0$, there exists $C_{\epsilon}>0$ so that

$$
\sum_{n:|n| \geq(1-\epsilon) \lambda_{j}}\left|\nu_{\lambda_{j}}^{x, \xi}(n)\right|^{2} \geq C_{\epsilon} .
$$

Lemma 3 implies Lemma 2 since it implies that for any $\epsilon>0$,

$$
\sum_{n:|n| \geq(1-\epsilon) \lambda_{j}}\left|\nu_{\lambda_{j}}^{x, \xi}(n)\right|^{2} e^{-2 n \tau} \geq C_{\epsilon} e^{2 \tau(1-\epsilon) \lambda_{j}} .
$$

In essence, we prove the lower bound in Proposition 1 in the ergodic case by showing that all of the Fourier coefficients in the allowed energy 
region $|n| \leq \lambda_{j}$ are of uniformly large size. Since the top frequency term dominates and its Fourier coefficient is large, $\gamma_{x, \xi}^{*} \varphi_{j}^{\mathbb{C}}$ must have maximal growth.

The argument sketched above only proves the desired logarithmic growth law of Proposition 1 for $L^{2}$-norms. Proposition 2 improves it to give the local $L^{1}$-convergence statement of Proposition 1.

The proof of Theorem 2 follows the same general outline but is more complicated for two reasons: first, the Fourier transform of $\gamma_{x, \xi}^{*} \varphi_{j}$ is the Fourier transform of an $L^{\infty}$ function and not an $L^{2}$ function. It can be shown that it is not even a measure and so we cannot speak of the 'size' of the Fourier coefficients. Hence it has to be multiplied by a decaying analytic function before the Fourier transform is taken. And as mentioned above, the Fourier coefficients may saturate the growth bounds somewhere on $\mathbb{R}$ for each $j$ but the location of the saturating mass may escape to infinity along the parameter interval. This explains why we may need to introduce translations $N_{j}$ into the times.

0.5. Generalizations. There are several natural generalizations of intersection problems for geodesics and nodal hypersurfaces to consider: (i) to general real analytic curves $C \subset M^{2}$ of a real analytic surface; (ii) to general real analytic hypersurfaces $H \subset M^{m}$ in any dimension; (iii) to generic or random geodesics in all dimensions. We plan to investigate the generalizations in a subsequent article.

The generalization (i) is simplest, since most of the techniques and results of this article apply to any real analytic curve $C \subset M^{2}$. The main one which does not is Proposition 2, in the cases when the curve is not a geodesic. Moreover, the unit speed parametrization of $C$ no longer complexifies to an isometric embedding of Grauert tubes, and it is not as simple to find the growth rate of $\frac{1}{\lambda_{j}} \log \mid \varphi_{j}^{\mathbb{C}}\left(\gamma_{C}^{\mathbb{C}}(t+i \tau) \mid\right.$; it does not equal $|\tau|$ when $C$ is not a geodesic, and one does not get the same concentration of complex zeros along the real points, i.e. there exists an additional smooth component to the limit distribution of complex zeros.

The additional complication in (ii) is that nodal hypersurfaces intersect other hypersurfaces $H$ in codimension 2 submanifolds rather than discrete points. Hence the limit measure will be a locally $L^{1}$ measure on a complex $(n-1)$-dimensional parameter domain. Instead of expanding the restriction as a Fourier series or integral we would need to use eigenfunctions of the hypersurface Laplacian.

The generalization (iii) is the most difficult, and it is not clear at the moment whether a generalization of Theorem 1 to periodic geodesics in higher dimensions exists. We cannot appeal to the QER result of [TZ] in this case. The QER result is a quantum analogue of the fact that unit (co-)vectors in $S_{H}^{*} M$, i.e. with footpoint on $H$, form a cross section to the geodesic flow when $H$ is a hypersurface and thus the first return 
map is ergodic. When $\operatorname{dim} H<\operatorname{dim} M-1, S_{H}^{*} M$ is no longer a cross section and the proof in $[\mathbf{T Z}]$ does not apply. It is not clear that the Fourier coefficients always have uniformly the same size for $|n| \leq \lambda$. It is tempting to relate the $L^{2}$ mass of restricted eigenfunctions to their global mass when the geodesic is Birkhoff regular (i.e. uniform) but the results so far do not improve on Theorem 2. However it is possible to prove a somewhat weaker version of Theorem 1 for random geodesics; we postpone the proof to a subsequent article.

0.6. Acknowledgements. Thanks to Simon Marshall for many helpful conversations on earlier drafts of this article, and to John Toth for collaboration on related problems in $[\mathbf{T Z}]$ and elsewhere.

\section{Geometry of geodesics and Grauert tubes}

In this section, we discuss the geometry of geodesics and their complexifications in Grauert tubes. We need to relate the holomorphic extension of $\gamma_{x, \xi}$ to the Hamilton flow of the Grauert tube function. The relations are valid in all dimensions and so we consider a general real analytic Riemannian manifold $M^{m}$ of dimension $m$.

1.1. Geodesic flow in the real domain. A potentially confusing point is that there are two geodesic flows in the real domain, and both are relevant to the microlocal analysis of wave groups. Below we denote by $\pi: T^{*} M \rightarrow M$ the standard projection.

- The geometer's geodesic flow: the Hamilton flow of the Hamiltonian $H(x, \xi)=|\xi|_{g}^{2}=\sum_{i, j=1}^{d} g^{i j}(x) \xi_{i} \xi_{j}$ on $T^{*} M$. We denote its flow by $g^{t}: T^{*} M \rightarrow T^{*} M$. The usual exponential map is defined by $\exp _{x} \xi=\pi g^{1}(x, \xi)$. On the zero section $0_{M} \subset T^{*} M$ it is the identity map.

- The homogeneous geodesic flow $G^{t}: T^{*} M-0 \rightarrow T^{*} M-0$ (the bicharacteristic flow of the wave operator): It is the Hamiltonian flow generated by $H_{1}(x, \xi)=|\xi|_{g}=\sqrt{H}$. It is homogeneous in the sense that $G^{t}(x, r \xi)=r G^{t}(x, \xi)$. It is not defined on $0_{M}$.

1.2. Grauert tubes. For background on Grauert tube geometry we refer to $[\mathbf{G S 1}, \mathbf{G S 2}, \mathbf{L S}]$. By a theorem of Bruhat-Whitney $[\mathbf{B W}]$, a real analytic manifold $M$ always possesses a complexification $M_{\mathbb{C}}$, i.e. a complex manifold of which $M$ is a totally real submanifold. A real analytic metric $g$ then determines a canonical plurisubharmonic function $\rho_{g}$ on $M_{\mathbb{C}}$ whose square root $\sqrt{\rho}$ is known as the Grauert tube function. In fact, $\rho(\zeta)=-\frac{1}{4} r_{\mathbb{C}}^{2}(\zeta, \bar{\zeta})$ where $r_{\mathbb{C}}^{2}$ is the holomorphic extension of the distance-squared function from a neighborhood of the diagonal in $M \times M$. The $(1,1)$ - form $\partial \bar{\partial} \sqrt{\rho}$ has rank $m-1$ on $M_{\epsilon} \backslash M$, and the leaves of its null foliation (the 'Monge-Ampère' or Riemann foliation) are the 
traces of the complexified geodesics $\gamma_{\mathbb{C}}(t+i \tau)$. The Grauert tubes are defined by

$$
M_{\epsilon}=\left\{\zeta \in M_{\mathbb{C}}: \sqrt{\rho}(\zeta) \leq \epsilon\right\} .
$$

Remark 1.1. We define $\sqrt{\rho}$ so that it measures the tube radius rather than the tube diameter, i.e. $\sqrt{\rho}(\zeta)=\frac{1}{2} \sqrt{-r_{\mathbb{C}}^{2}(\zeta, \bar{\zeta})}$. Thus on a flat torus, $\sqrt{\rho}(x+i \xi)=|\xi|$.

We define the Kähler form $\omega=\omega_{g}$ of $M_{\epsilon}$ by

$$
\omega=i^{-1} \partial \bar{\partial} \rho .
$$

The unusual sign convention (making the Kähler form a negative rather than positive) $(1,1)$ form) is adopted from [GS1]. In terms of the real operators (with $J^{*}$ the complex structure operator on 1 -forms),

$$
d^{c}=\frac{i}{4 \pi}(\bar{\partial}-\partial)=-\frac{1}{4 \pi} J^{*} d, \quad d d^{c}=-d^{c} d=\frac{i}{2 \pi} \partial \bar{\partial},
$$

we have

$$
\omega=-2 \pi d d^{c} \rho .
$$

Following [GLS], we define the imaginary time complexified exponential map by

$$
E:(x, \xi) \in B_{\epsilon}^{*} M \rightarrow \exp _{x}^{\mathbb{C}} \sqrt{-1} \xi \in M_{\epsilon} .
$$

The following Lemma records the way that $E$ transfers objects between $M_{\epsilon}$ and the co-ball bundle $B_{\epsilon}^{*} M$ of radius $\epsilon$ in $T^{*} M$.

Lemma 1.2. Let $\alpha=\xi \cdot d x$ resp. $\omega_{T^{*} M}$ be the canonical 1-form, resp. symplectic form, of $T^{*} M$. Then $E:\left(B_{\epsilon}^{*} M, \omega_{T^{*} M}\right) \rightarrow\left(M_{\epsilon}, \omega\right)$ is a symplectic diffeomorphism from the co-ball bundle of radius $\epsilon$ to $M_{\epsilon}$. It has the properties:

- $E^{*}|\xi|_{g}^{2}=\rho_{g}$ and $E^{*}|\xi|_{g}=\sqrt{\rho}$.

- $E^{*} \alpha=\Im \bar{\partial} \rho=d^{c} \sqrt{\rho}$ and $E^{*} \omega=\omega_{T^{*} M}$.

Proof. This is a reformulation of some results of [GS1, GLS, LS]. The Theorem on p. 568 of [GS1] states that $\alpha=\Im \bar{\partial}|\xi|_{g}^{2}$ on $T^{*} M$ where $\bar{\partial}$ is with respect to the "adapted complex structure" on $B_{\epsilon}^{*} M$. Using a theorem of Kostant-Sternberg, they produce a unique diffeomorphism $\psi:\left(B_{\epsilon}^{*} M, \omega_{T^{*} M}\right) \rightarrow\left(M_{\epsilon}, \omega\right)$. It thus suffices to show that $E=\psi$. This follows from the uniquness argument in [GLS] Proposition 1.7 and Theorem 1.8. That is, $\psi \circ E^{-1}$ is a biholomorphic map of $M_{\epsilon}$ which equals the identity on the totally real submanifold $M \subset M_{\epsilon}$, and therefore must be the identity map.

q.e.d. 
Since $\left(M_{\tau}, \omega_{\rho}\right)$ is a symplectic manifold, we can consider Hamiltonian flows of $\rho$ and $\sqrt{\rho}$. We denote the Hamilton flow of any function $H$ with respect to $\omega=\omega_{\rho}$ by $\exp t \Xi_{H}^{\omega}$. When $\omega$ is understood, we simply denote by $\Xi_{\sqrt{\rho}}$ the Hamilton vector field of $\sqrt{\rho}$ with respect to $\omega$. The following Lemma asserts that $E$ interwines (both) geodesic flows on $B_{\epsilon}^{*} M-0$ with Hamilton flows on $M_{\epsilon}$.

\section{Lemma 1.3.}

$$
E \circ G^{t}=\exp t \Xi_{\sqrt{\rho}} \circ E, \quad E \circ g^{t}=\exp t \Xi_{\rho} \circ E .
$$

Proof. It follows from Lemma 1.2 that $E^{*} \sqrt{\rho}=|\xi|$ and that $E^{*} \omega=$ $\omega_{B^{*} M}$. Hence $E$ intertwines the associated Hamiltonian flows. Similarly for the Hamiltonian flow of $\rho$.

q.e.d.

We recall that the Liouville measure on each sphere bundle $S_{\epsilon}^{*} M$ of radius $\epsilon$ is given by $d \mu_{L}=\alpha \wedge \omega_{T^{*} M}^{d-1}$. Under the complexified exponential map $E$, Liouville measure pulls to $\partial M_{\epsilon}$ to

$$
E^{*} d \mu_{L}=d^{c} \sqrt{\rho} \wedge \omega^{m-1} \text {. }
$$

1.3. Complexified Hamiltonian flow. In addition to its Kähler form $\omega_{g} \in \Omega^{1,1}\left(M_{\mathbb{C}}\right)$, the Grauert tube carries the complex holomorphic metric

$$
g_{\mathbb{C}}=\sum_{i, j=1}^{n} g^{i j}(\zeta) d \zeta_{i} \otimes d \zeta_{j}
$$

in local holomorphic coordinates on $M_{\mathbb{C}}$. The complexified geodesic flow

$$
g_{\mathbb{C}}^{t+i \tau}: T^{*} M_{\mathbb{C}} \rightarrow T^{*} M_{\mathbb{C}}
$$

is the partially defined Hamiltonian flow of the holomorphic Hamiltonian,

$$
H_{\mathbb{C}}(\zeta, \xi)=\sum_{i, j=1}^{n} g^{i j}(\zeta) \xi_{i} \xi_{j}
$$

on $T^{*}\left(M_{\mathbb{C}}\right)$ with respect to its holomorphic symplectic form

$$
\sigma=\sum_{j} d \zeta_{k} \wedge d \xi_{k}
$$

where $\xi_{k}$ are the coordinates of a $(1,0)$-form in the basis $d \zeta_{k}$. The Hamiltonian vector field is then

$$
\Xi_{H}=\sum_{i, j=1}^{n} g^{i j}(\zeta) \xi_{i} \frac{\partial}{\partial \zeta_{j}}-\sum_{i, j=1}^{n} \frac{\partial g^{i j}(\zeta)}{\partial \zeta_{k}} \xi_{i} \xi_{j} \frac{\partial}{\partial \xi_{k}} .
$$

In these coordinates the canonical one-form on $T^{*} M_{\mathbb{C}}$ is given by

$$
\alpha_{T^{*} M_{\mathbb{C}}}=\sum_{j} \xi_{j} d \zeta_{j}
$$


We also denote by $\pi: T^{*} M_{\mathbb{C}} \rightarrow M_{\mathbb{C}}$ the natural projection.

The following Lemma relates $\gamma_{x, \xi}^{\mathbb{C}}(t+i \tau)$ to the holomorphic geodesic flow.

Lemma 1.4. Let $(x, \xi) \in T^{*} M$ and let $\gamma_{x, \xi}(t+i \tau): \mathbb{R} \rightarrow M_{\tau}$ be the analytic continuation of $\gamma_{x, \xi}(t)=\exp _{x} t \xi$. Then:

- $\gamma_{x, \xi}(t+i \tau)=\pi g_{\mathbb{C}}^{t+i \tau}(x, \xi)$.

- $E(x, \xi)=\pi g_{\mathbb{C}}^{1}(x, i \xi)$.

- $g_{\mathbb{C}}^{t+i \tau}=g_{\mathbb{C}}^{i \tau} g_{\mathbb{C}}^{t}$.

- $\gamma_{x, \xi}(t+i \tau)=\pi G^{t+i \tau}(x, \xi)=\pi G^{i \tau} G^{t}(x, \xi)=\gamma_{G^{t}(x, \xi)}(i \tau)$.

Indeed, these identities hold for real $t$ and therefore analytically continue to complex $t$.

1.4. Hamiltonian flow of $\sqrt{\rho}$ and complexified geodesics. In this section we relate $\gamma_{x, \xi}^{\mathbb{C}}(t+i \tau)$ to the Hamilton orbits of $\Xi_{\sqrt{\rho}}$. Although both arise from Hamilton flows of metric functions, it is not obvious that the holomorphic configuration space orbits $\gamma_{x, \xi}^{\mathbb{C}}(t+i \tau)$ should be the same curves for fixed $\tau$ as the 'phase space' Hamilton orbits $\exp t \Xi_{\sqrt{\rho}}$ on $\partial M_{\tau}$. We now prove that indeed they are the same curves.

As a (simple) example we consider the flat torus $\mathbb{R}^{n} / \mathbb{Z}^{n}$. In this case $E(x, \xi)=x+i \xi$ and

$$
\left\{\begin{array}{l}
\gamma_{x, \xi}(t)=x+t \frac{\xi}{|\xi|}, \quad \gamma_{x, \xi}(t+i \tau)=x+(t+i \tau) \frac{\xi}{|\xi|}, \\
\exp t \Xi_{\sqrt{\rho}}(x+i \xi)=E\left(x+t \frac{\xi}{|\xi|}, \xi\right)=x+(t+i \tau) \frac{\xi}{|\xi|} .
\end{array}\right.
$$

In the last line, we assume that $\sqrt{\rho}(E(x, \xi))=|\xi|=\tau$.

Another way to contrast the two flows is the following: $\partial M_{\tau}$ is a contact co-isotropic manifold for $\omega_{\rho}$ and the flow lines of the Hamilton flow of $\sqrt{\rho}$ or of $\rho$ form its real one dimensional null-foliation. On the other hand, $d d^{c} \sqrt{\rho}$ has a complex one dimensional null foliation on $M_{\tau}$. We wish to relate the real null-foliation for $\omega_{\rho}$ on $\partial M_{\tau}$ to the holomorphic null foliation for $d d^{c} \sqrt{\rho}$ on all of $M_{\tau}$.

Proposition 1.5. The orbit of the Hamiltonian flow of $\sqrt{\rho}$ through $\gamma_{x, \xi}(i \tau)$ on $\partial M_{\tau}$ is the curve $t \rightarrow \gamma_{x, \xi}(t+i \tau)$. That is, $\exp t \Xi_{\sqrt{\rho}}(E(x, \xi))$ $=\gamma_{x, \xi}(t+i|\xi|)$.

Proof. Let $(x, \xi) \in T^{*} M$ be a real co-vector. It follows from Lemma 1.3 that $\exp t \Xi_{\sqrt{\rho}}(E(x, \xi))=E \circ G^{t}(x, \xi)$ and then by Lemma 1.4 that

$$
\pi G_{\mathbb{C}}^{t+i}(x, \xi)=\pi G_{\mathbb{C}}^{i} G_{\mathbb{C}}^{t}(x, \xi)=E\left(G^{t}(x, \xi)\right)=\exp t \Xi_{\sqrt{\rho}}(E(x, \xi)) .
$$

Also, we have ([GLS $])$

$$
\exp _{\gamma(t)} i s \dot{\gamma}(t)=\gamma(t+i s) \text {. }
$$

Indeed, let $\beta(r)$ be the geodesic with initial conditions $\beta(0), \dot{\beta}(0)=$ $(\gamma(t), s \dot{\gamma}(t))$. By definition, $\exp _{\gamma(t)} i s \dot{\gamma}(t)$ is the analytic continuation of 
$r \rightarrow \beta(r)$ at $r=i$. But $\beta(r)=\gamma(t+r s)$ for real $r$ so $\beta(i)=\gamma(t+i s)$.

Therefore $(27)$ holds. Since $E(x, \xi)=\exp _{x} i \xi$ this says,

$$
E(\gamma(t), s \dot{\gamma}(t))=\gamma(t+i s) .
$$

Putting $s=|\xi|$ gives

$$
E\left(G^{t}(x, \xi)\right)=\gamma_{x, \xi}(t+i|\xi|) .
$$

Combining (26) and (28) we have

$$
\exp t \Xi_{\sqrt{\rho}}(E(x, \xi))=E \circ G^{t}(x, \xi)=\gamma_{x, \xi}(t+i|\xi|),
$$

completing the proof.

q.e.d.

The following Corollary is also in $[\mathbf{L S}]$.

Corollary 1.6. $\gamma_{x, \xi}(i \tau)$ is a flow line of the gradient field $\nabla \sqrt{\rho}$.

1.5. Kähler isometric embedding of geodesics. As in the introduction, we let (2) be an arc-length parametrized geodesic, and let (3) denote its analytic extension to a strip. The special properties of geodesics are given in the following

Proposition 1.7. The map $\gamma_{x, \xi}: S_{\epsilon} \rightarrow M_{\epsilon}$ is a Kähler isometric embedding. More precisely,

1) $\rho\left(\gamma_{x, \xi}(t+i \tau)\right)=\tau^{2}$

2) $\sqrt{\rho}\left(\gamma_{x, \xi}(t+i \tau)=|\tau|\right.$;

3) $\gamma_{x, \xi}^{*} d d^{c} \rho=d t \wedge d \tau$

4) $\gamma_{x, \xi}^{*} d d^{c} \sqrt{\rho}=\delta_{0}(\tau) d t d \tau$.

Proof. Since $\gamma_{x, \xi}$ is holomorphic, the last two statements follow from the first two, which are equivalent. The first statement follows from the fact that the holomorphic extension of $\gamma_{x, \xi}$ corresponds under $E$ (19) to the homogeneous lift of $\gamma_{x, \xi}$ to $T^{*} M$, i.e.

$$
\tilde{\gamma}_{x, \xi}(t+i \tau)=\tau \gamma_{x, \xi}^{\prime}(t): S_{\epsilon} \rightarrow B^{*} M
$$

and the holomorphicity of this map is essentially the definition of the adapted complex structure in [LS, GS1]. Thus (as in Proposition 1.5), $\gamma_{x, \xi}(t+i \tau)=E\left(\gamma_{x, \xi}(t), \tau \gamma_{x, \xi}^{\prime}(t)\right)$.

By [GLS] (p. 686), with $d=r_{\mathbb{C}}$,

$$
d^{2}\left(q, \exp _{q} i \xi\right)=-|\xi|_{q}^{2}
$$

But

$$
\sqrt{\rho}\left(\exp _{q} i \xi\right)=\frac{1}{2} \sqrt{-d^{2}\left(\exp _{q} i \xi, \exp _{q}-i \xi\right)}=\sqrt{-d^{2}\left(0, \exp _{q} i \xi\right)}
$$

by analytic extension of the equation $d^{2}\left(\exp _{q} \xi, \exp _{q}-\xi\right)=4 d\left(q, \exp _{q} \xi\right)$. Thus, 
ERGODICITY \& INTERSECTIONS OF NODAL SETS AND GEODESICS 319

$$
-\rho\left(\gamma_{x, \xi}(t+i \tau)\right)=\frac{1}{4} d^{2}\left(\gamma_{G^{t}(x, \xi)}(i \tau), \gamma_{G^{t}(x, \xi)}(-i \tau)\right)=-\tau^{2} .
$$

q.e.d.

\section{Szegö kernels on boundaries of Grauert tubes}

The proofs of Proposition 2 and Proposition 2 are based on microlocal analysis in the complex domain. In this section and the next, we introduce the basic objects of microlocal analysis on Grauert tubes: the Szegö projectors and Poisson extension operator. To the extent possible, we refer to $[\mathbf{B o G u}, \mathbf{B o S j}, \mathbf{G S 2}, \mathbf{Z}]$ for background. We include further background on Fourier integral operators with complex phase in the Appendix $\S 10$.

As in $[\mathbf{Z}]$, we study the analytic continuation of eigenfunctions via the Poisson operator

$$
P^{\tau}: L^{2}(M) \rightarrow \mathcal{O}^{0}\left(\partial M_{\tau}\right), \quad P^{\tau}(\zeta, y)=\sum_{j} e^{-\tau \lambda_{j}} \varphi_{j}^{\mathbb{C}}(\zeta) \varphi_{j}(y),
$$

which is defined by analytically continuing the Schwartz kernel of the Poisson semi-group

$$
U^{i \tau}=e^{-\tau \sqrt{\Delta}}: L^{2}(M) \rightarrow L^{2}(M)
$$

in the first variable to $\zeta \in M_{\tau}$ and then restricting it to $\partial M_{\tau}$. As reviewed in $\S 2-\S 3, \mathcal{O}^{0}\left(\partial M_{\tau}\right)$ denotes the Hilbert space of boundary values of holomorphic functions in $M_{\tau}$ which belong to $L^{2}\left(\partial M_{\tau}\right)$. The Poisson operator is a Fourier integral operator with complex phase and its wave front is contained in the graph of the complexified geodesic flow (see $\S 3)$.

2.1. Szegö projector on boundaries of Grauert tubes. We denote by $\mathcal{O}^{s+\frac{m-1}{4}}\left(\partial M_{\epsilon}\right)$ the subspace of the Sobolev space $W^{s+\frac{m-1}{4}}\left(\partial M_{\epsilon}\right)$ consisting of CR holomorphic functions, i.e.

$$
\mathcal{O}^{s+\frac{m-1}{4}}\left(\partial M_{\epsilon}\right)=W^{s+\frac{m-1}{4}}\left(\partial M_{\epsilon}\right) \cap \mathcal{O}\left(\partial M_{\epsilon}\right) .
$$

The inner product on $\mathcal{O}^{0}\left(\partial M_{\epsilon}\right)$ is with respect to the Liouville measure $d \mu_{\epsilon}$.

For each $\tau<\epsilon$, the Szegö projector

$$
\Pi_{\tau}: L^{2}\left(\partial M_{\tau}\right) \rightarrow \mathcal{O}^{0}\left(\partial M_{\tau}\right)
$$

of the tube $M_{\tau}$ is the orthogonal projection onto boundary values of holomorphic functions in the tube. It is proved by Boutet de MonvelSjöstrand $[\mathbf{B o S j}]$ that $\Pi_{\tau}$ is a complex Fourier integral operator, whose real canonical relation is the graph $\Delta_{\Sigma_{\tau}}$ of the identity map on the symplectic cone $\Sigma_{\epsilon} \subset T^{*}\left(\partial M_{\tau}\right)$ spanned by the contact form $\alpha_{\tau}:=$ $\left.d^{c} \rho\right|_{\left(\partial M_{\tau}\right)}$, i.e.

$$
\Sigma_{\tau}=\left\{\left(x, \xi ; r \alpha_{\epsilon}\right), \quad(x, \xi) \in \partial M_{\tau}, r>0\right\} \subset T^{*}\left(\partial M_{\tau}\right) .
$$


This symplectic cone is symplectically equivalent to $T^{*} M$ under the homogeneous map

$$
\iota_{r}: T^{*} M-0 \rightarrow \Sigma_{\tau}, \quad \iota_{\epsilon}(x, \xi)=\left(x, \tau \frac{\xi}{|\xi|},|\xi| \alpha_{\left(x, \tau \frac{\xi}{|\xi|}\right)}\right) .
$$

The parametrix construction of $[\mathbf{B o S j}]$ for Szegö kernels of strictly pseudo-convex domains applies to $\Pi_{\tau}$ and has the form

$$
\Pi_{\tau}(x, y)=\int_{0}^{\infty} e^{i \theta \psi(x, y)} A(x, y, \theta) d \theta,
$$

where $\psi(x, y)=\tau^{2}-\rho(x, y)$ and where $A$ is a classical symbol of order $2 m-1$ in $\theta$.

In the theory of Fourier integral operators with complex phase of [MSj], the full complex canonical relation of $\Pi_{\tau}$ lies in the (Cartesian square of the) complexification $\widetilde{\partial M}_{\tau}$ of $\partial M_{\tau}$. In general, the canonical relations associated to the Schwartz kernels $K(x, y)$ of Fourier integral operators with complex phase lie in the complexification of the relevant cotangent bundles $T^{*}\left(X \times X^{\prime}\right)$. But the wave front set $W F(K)$ is contained in the real points of the canonical relation. Since the symplectic geometry in the real domain is simpler, we choose to work in the framework of adapted Fourier integral operators and Toeplitz operators of [BoGu] (Section A.2 of the Appendix; see Definition 2.7). In the theory of Toeplitz operators of [BoGu], a special symbol calculus is defined for a sub-class of Fourier integral operators with complex phase known as Toeplitz operators or Hermite Fourier integral operators (see $\S 3$ of $[\mathbf{B o G u}]$ for the definition). The Toeplitz calculus of $[\mathbf{B o G u}]$ applies to the operators in our problem, and it is not necessary for us to analytically continue to the complexification $\left(\partial M_{\tau}\right)^{\mathbb{C}}$.

We briefly recall the definition of adapted Fourier integral operators. Let $X, X^{\prime}$ be two real $C^{\infty}$ symplectic manifolds and let $\Sigma \subset T^{*} X, \Sigma^{\prime} \subset$ $T^{*} X^{\prime}$ be two symplectic subcones. Let $\chi: \Sigma \rightarrow \Sigma^{\prime}$ be a homogeneous symplectic isomorphism, i.e one that commutes with the $\mathbb{R}_{+}$action on the cones. Then a Fourier integral operator with complex phase from $X$ to $X^{\prime}$ is said to be adapted to $\chi$ if its complex canonical relation $C$ is positive, and if its real part $C_{\mathbb{R}}$ is the graph of $\chi$. It is called elliptic if its symbol is nowhere vanishing. In this language, $\Pi_{\tau}$ is a Fourier integral operator with complex phase which is adapted to the identity map $\chi=I d_{\Sigma_{\tau}}: \Sigma_{\tau} \rightarrow \Sigma_{\tau}$. For background on general complex canonical relations and their real points, we refer to $[\mathbf{M S j}]$ (see also $\S 10$ ).

As discussed in the Appendix of [BoGu] (section (2.11)), a Fourier integral operator $A$ with complex phase adapted to $\chi$ is a Hermite operator. The authors define the symbol at any point of the graph of $\chi$ to be a half-density on the graph of $\chi$ tensored with a linear operator

$$
K_{\sigma_{A}} f(u)=\lambda \int e^{-q(u, v)} f(v) d v
$$


on $\mathcal{S}\left(\mathbb{R}^{n}\right)$ where $\lambda \in \mathbb{C}$ and $q(u, v)$ is a quadratic form with positive real part. Here, $\mathbb{R}^{n}$ is the symplectic orthogonal complement in $T\left(T^{*} \partial M_{\tau}\right)$ to $T \Sigma$. It is shown in [BoGu] that the principal symbol of any Szegö projector $\Pi_{\tau}$ is a rank one projector onto the 'ground state' annihilated by the Lagrangian system associated to the $\bar{\partial}$ operator. By comparison, in the symbol calculus of $[\mathbf{M S} \mathbf{j}]$, the symbol is a half-density on the complex canonical relation.

\section{Poisson kernels and their analytic continuations}

In this section we study the Poisson operator $P^{\tau}$ (30) and its composition with the wave group $U^{t}=e^{i t \sqrt{\Delta}}$. We begin by reviewing the proof of the following

Proposition 3.1. Let $(M, g)$ be a real analytic compact Riemannian $m$-manifold. Then $P^{\tau} U^{t}$ is a Fourier integral operator with complex phase of order $-\frac{m-1}{4}$ associated to the positive complex canonical relation

$$
\mathcal{G}_{\tau, t}=\left\{\left(y, \eta, \zeta, p_{\zeta}\right\} \subset T^{*} \widetilde{M} \times T^{*} \widetilde{\partial M}_{\tau}:\left(\zeta, p_{\zeta}\right) \sim G^{i t+t}(y, \eta)\right\}
$$

where $\sim$ denotes the equivalence relation in (91) of $\S 10$.

Here, $\widetilde{M}=M_{\mathbb{C}}$ denotes the complexification of $M$. Since $\operatorname{dim}_{\mathbb{C}} \widetilde{M}=$ $m, \operatorname{dim}_{\mathbb{C}} \widetilde{\partial M}_{\tau}=2 m-1$ and so the canonical relation has complex dimension $3 m-1$.

As mentioned above, it is simpler to work in the framework of adapted Fourier integral operators, since the symbols live on the real points of the canonical relation. In the following Proposition, we use an extension of the notion of adapted Fourier integral operator in the sense of $\S 2$. Namely, we allow the homogeneous symplectic map to be a symplectic embedding rather than a symplectic isomorphism. All of the composition results of $[\mathbf{B o G u}]$ extend readily to this case.

Proposition 3.2. $P^{\tau}$ is a Fourier integral operator with complex phase of order $-\frac{m-1}{4}$ adapted to the isomorphism $\iota_{\tau}: T^{*} M-0 \rightarrow \Sigma_{\tau}$ (32). Moreover, for any $s$,

$$
P^{\tau}: W^{s}(M) \rightarrow \mathcal{O}^{s+\frac{m-1}{4}}\left(\partial M_{\tau}\right)
$$

is a continuous isomorphism.

This Proposition is proved in $[\mathbf{Z 2}, \mathbf{S t}]$. A somewhat less precise statement is given in [Bou] and in Theorem 3.2 of [GS2], but the proof is hardly indicated there. Since the statement and proof of Proposition 3.2 differ somewhat from the previous versions, we review its proof. We also need the following extension: 
Proposition 3.3. $P^{\tau} \circ U^{t}$ is a Fourier integral operator with complex phase of order $-\frac{m-1}{4}$ adapted to the symplectic isomorphism

$$
\chi_{\tau, t}(y, \eta)=\iota_{\tau}\left(G^{t}(y, \eta): T^{*} M-0 \rightarrow \Sigma_{\tau} .\right.
$$

Equivalently, $P^{\tau} \circ U^{t}$ is a Fourier integral operator of Hermite type of order $-\frac{m-1}{4}$ associated to the canonical relation

$$
\Gamma_{\tau, t}=\left\{\left(\iota_{\tau}\left(G^{t}(y, \eta)\right), y, \eta\right)\right\} \subset \Sigma_{\tau} \times T^{*} M .
$$

Proof. This follows immediately from Proposition 3.2 and from the fact proved in $[\mathbf{B o G u}]$, Theorems 3.4 and 7.5, that the composition of a Fourier integral operator and a Fourier integral operator of Hermite type is also a Fourier integral operator of Hermite type, with a certain addition law for the orders and a composition law for the symbols.

q.e.d.

The proofs of Proposition 3.2-3.3 can be extracted from Hadamard's classical construction of a convergent parametrix for the Schwartz kernels of the operators $\cos t \sqrt{\Delta}$ and $\frac{\sin t \sqrt{\Delta}}{\sqrt{\Delta}}$ in a small neighborhood of the characteristic conoid. Hadamard did not consider the operator $\exp (i t \sqrt{\Delta})$ since $\sqrt{\Delta}$ was unknown at that time, but in $[\mathbf{Z 2}]$ we modify the Hadamard parametrix construction to construct the Schwartz kernel $U(t, x, y)$ of $U^{t}$ as a convergent Riesz series expansion near the characteristic conoid. Further it is shown that $U(i \tau, x, y)$ admits an analytic continuation in $x$ when $(i \tau, x, y)$ lies in a small enough neighborhood of the complex characteristic conoid. Outside of this neighborhood $U(i \tau, x, y)$ is real analytic. It follows from the construction that $U(i \tau)$ is a Fourier integral operator of complex type, and its canonical relation is the graph of the imaginary time geodesic flow $G^{i \tau}$; we refer to $[\mathbf{Z 2}]$ for the details.

However, we need one additional statement to justify the claim that $P^{\tau}$ is adapted to $\iota_{\tau}$. The canonical relation $\mathcal{G}_{\tau, t}$ of Proposition 3.2 is the holomorphic extension of $G^{t}$. We need to see that the intersection of this graph with $\Sigma_{\tau} \times T^{*} M$ is $\Gamma_{\tau, t}$. The proof involves the same identifications as in $\S 1.4$.

We recall that $G^{t}: T^{*} M-0 \rightarrow T^{*} M-0$ is the homogeneous geodesic flow, i.e. $G^{t}(x, \xi)=|\xi| G^{t}\left(x, \frac{\xi}{|\xi|}\right)$. The analytic continuation in $t$ is also homogeneous, so we have

$$
G^{i \tau}(x, \xi)=|\xi| G^{i \tau}\left(x, \frac{\xi}{|\xi|}\right) .
$$

Lemma 3.4. We have: $\iota_{\tau}(y, \eta)=G^{i \tau}(y, \eta)$. Thus, $G^{i \tau}$ gives a homogeneous symplectic isomorphism

$$
G^{i \tau}: T^{*} M-0 \rightarrow \Sigma_{\tau}-0
$$


Proof. By homogeneity we may assume that $|\xi|=1$. We then want to show that

$$
G^{i \tau}(x, \xi)=\left(\exp _{x} i \tau \xi, \alpha_{\tau}\right)
$$

where $\alpha_{\tau}$ is the canonical one form of $T_{\exp _{x} i \tau \xi} \partial M_{\tau}$. Note that $G^{t}(x, \xi)=$ $g^{t}(x, \xi)$ for $|\xi|=1$ and we can analytically continue $g^{t}(x, \xi)$ in $t$. By definition, for $(x, \xi) \in S^{*} M \subset T^{*} M_{\mathbb{C}}$,

$$
g^{i \tau}(x, \xi) \in T_{\gamma_{x, \xi}(i \tau)}^{*} M_{\tau}
$$

is defined by extending the phase space Hamilton orbit $\tilde{\gamma}_{x, \xi}: \mathbb{R} \rightarrow$ $T^{*} M$ holomorphically in time as an orbit of flow of the holomorphic Hamiltonian (23) and for fixed $(x, \xi), \tilde{\gamma}_{x, \xi}$ is a holomorphic strip in $T^{*} M_{\mathbb{C}}$. We then restrict it to the imaginary time axis to obtain a real curve

$$
\tilde{\gamma}_{x, \xi}(i \tau):\left[0, \epsilon_{0}\right) \rightarrow T^{*} M_{\mathbb{C}} .
$$

If $\pi_{\mathbb{C}}: T^{*} M_{\mathbb{C}} \rightarrow M_{\mathbb{C}}$ denotes the natural projection, then $\pi_{\mathbb{C}}\left(\tilde{\gamma}_{x, \xi}(i \tau)\right)=$ $\gamma_{x, \xi}(i \tau)$, so the only non-obvious aspect is the $p_{\zeta}$ component. But $\tilde{\gamma}_{x, \xi}(i \tau)$ is the cotangent lift of $\gamma_{x, \xi}(i \tau)$, hence is given by

$$
G^{i \tau}(x, \xi)=\left(\gamma_{x, \xi}(i \tau), g_{\mathbb{C}} \frac{d}{d \tau} \gamma_{x, \xi}(i \tau)\right)
$$

where $g_{\mathbb{C}}: T M_{\mathbb{C}} \rightarrow T^{*} M_{\mathbb{C}}$ is the linear map defined by the analytic continuation (21) of the Riemannian metric to a holomorphic symmetric two tensor on $T M_{\mathbb{C}}$. The formula (40) is the analytic continuation of the lifting formula in the real domain. It remains to prove that

$$
\left(\gamma_{x, \xi}(i \tau), g_{\mathbb{C}} \frac{d}{d \tau} \gamma_{x, \xi}(i \tau)\right)=\alpha_{\left.\gamma_{x, \xi}(i \tau)\right)} .
$$

We recall that $\frac{d}{d \tau} \gamma_{x, \xi}(i \tau) \in T_{\gamma_{x, \xi}(i \tau)} M_{\mathbb{C}}$ and that $\gamma_{x, \xi}(i \tau) \in \partial M_{\tau}$. The curve $t \rightarrow \gamma_{x, \xi}(t+i \tau)$ is characteristic for the form $\omega$ restricted to $\partial M_{\tau}$, and so $\frac{d}{d t} \gamma_{x, \xi}(t+i \tau)$ spans the null space of the form. Note that $\frac{d}{d \tau} \gamma_{x, \xi}(i \tau)=\left.i \frac{d}{d t} \gamma_{x, \xi}(t+i \tau)\right|_{t=0}$ and that $T:=\left.\frac{d}{d t} \gamma_{x, \xi}(t+i \tau)\right|_{t=0}$ and $\frac{d}{d \tau} \gamma_{x, \xi}(i \tau)=J T$ are symplectically paired by $\omega_{\gamma_{x, \xi}(i \tau)}$.

We now dualize to $T^{*} \partial M_{\tau}$ using the metric. We let $\alpha_{\tau}$ denote the canonical 1-form of $T^{*} \partial M_{\tau}$. Then $\alpha_{\tau}(T)=1$ and $\operatorname{ker} \alpha_{\tau}$ is the CR sub-bundle of $T \partial M_{\tau}$. The claim (41) is equivalent to

$$
g_{\mathbb{C}}\left(\frac{d}{d \tau} \gamma_{x, \xi}(i \tau), \cdot\right)=\omega(J T, \cdot)=\alpha_{\gamma_{x, \xi}(i \tau)}(\cdot) \in T_{\gamma_{x, \xi}(i \tau)} M_{\tau} .
$$

Indeed, we note that we get 1 if we evaluate both sides on $T$, and so it suffices to check that we get 0 on the CR sub-bundle. But the CR subbundle is $J$-invariant and symplectically orthogonal to $T$, and therefore also to $J T$. 
Alternatively, we compute using Hamilton's equations (24). If $G^{i \tau}(x, \xi)=(\zeta, p)$, we have

$$
\begin{aligned}
g_{\mathbb{C}} \frac{d}{d \tau} \gamma_{x, \xi}(i \tau) & =g_{\mathbb{C}}\left(\sum_{i, j} g^{i j}\left(\gamma_{x, \xi}(i \tau)\right) p_{i} \frac{\partial}{\partial \zeta_{j}}\right. \\
& =\left(\sum_{i, j, k} g^{i j}\left(\gamma_{x, \xi}(i \tau)\right) p_{i} g_{j k}\left(\gamma_{x, \xi}(i \tau)\right) d \zeta_{k}\right. \\
& =\sum_{k} p_{k} d \zeta_{k}=\alpha_{\left(\gamma_{x, \xi}(i \tau)\right)} .
\end{aligned}
$$

Here, we also use that $\left.\alpha_{\mathbb{C}}\right|_{T \Sigma}=\alpha_{\Sigma}$. In other words, if we restrict the holomorphic canonical one form to $T \Sigma$, then it agrees with the restriction of the canonical one form of $T^{*}\left(\partial M_{\tau}\right)$ to $T \Sigma$. But this is obvious since $\alpha_{\mathbb{C}}$ is the analytic continuation of the canonical 1-form of $T^{*} \partial M_{\tau}$.

q.e.d.

3.1. Poisson kernel and Szegö kernel. Define the self-adjoint operator on $L^{2}(M, d V)$ by

$$
A_{\tau}=\left(P^{\tau *} P^{\tau}\right)^{-\frac{1}{2}}
$$

By Lemma 3.1 of $[\mathbf{Z}] A_{\tau}$ is an elliptic self-adjoint pseudo-differential operator of order $\frac{m-1}{4}$ with principal symbol $|\xi|^{\frac{m-1}{4}}$. Equivalently, $P^{\tau *} P^{\tau}$ is a pseudo-differential operator of order $-\frac{m-1}{2}$ with principal symbol $|\xi|^{-\frac{m-1}{2}}$.

Proposition 3.5. The Szegö kernel $\Pi_{\tau}$ is related to the Poisson kernel $P^{\tau}$ by

$$
\Pi_{\tau}=P^{\tau} A_{\tau}^{2} P^{\tau *} .
$$

Proof. Let $\tilde{\Pi}_{\tau}$ denote the operator in the statement. It is easily verified that

$$
\tilde{\Pi}_{\tau}^{*}=\tilde{\Pi}_{\tau}, \quad \tilde{\Pi}_{\tau} \tilde{\Pi}_{\tau}=\tilde{\Pi}_{\tau} .
$$

To check the idempotent property we note that

$$
P^{\tau}\left(P^{\tau *} P^{\tau}\right)^{-1} P^{\tau *} P^{\tau}\left(P^{\tau *} P^{\tau}\right)^{-1} P^{\tau *}=P^{\tau}\left(P^{\tau *} P^{\tau}\right)^{-1} P^{\tau *} .
$$

Further the range of $\tilde{\Pi}_{\tau}$ equals $\mathcal{O}^{0}\left(\partial M_{\tau}\right)$. Thus it is a projection onto that space. Moreover it is the orthogonal projection since it annihlates any $f \perp \mathcal{O}^{0}\left(\partial M_{\tau}\right)$ : Indeed, $\mathcal{O}^{0}\left(\partial M_{\tau}\right)$ is the image of $P^{\tau}$ on $L^{2}(M)$ and $P_{\tau}^{*} f=0$ if $f \perp \mathcal{O}^{0}\left(\partial M_{\tau}\right)$.

q.e.d.

Proposition 3.6. The operator $P^{\tau} A_{\tau}$ is a unitary operator: $L^{2}(M) \rightarrow \mathcal{O}^{0}\left(\partial M_{\tau}\right)$ with left inverse $A_{\tau} P^{\tau *}$. Moreover, $A_{\tau}^{2} P^{\tau *}$ : $\mathcal{O}^{0}\left(\partial M_{\tau}\right) \rightarrow L^{2}(M)$ is a left inverse to $P_{\tau}$. 
Proof. It suffices to show that

$$
\left\{\begin{array}{l}
A_{\tau} P^{\tau *} P^{\tau} A_{\tau}=A_{\tau}^{2} P^{\tau *} P^{\tau}=I d: L^{2}(M) \rightarrow L^{2}(M), \\
P^{\tau} A_{\tau}^{2} P^{\tau *}=\Pi_{\tau}: \mathcal{O}^{0}\left(\partial M_{\tau}\right) \rightarrow \mathcal{O}^{0}\left(\partial M_{\tau}\right) .
\end{array}\right.
$$

The identities are obvious (see Proposition 3.5).

We note that the CR functions $\left\{u_{j}^{\tau}\right\}$ are not in general orthogonal. However, it follows from Proposition 3.6 that

Corollary 3.7. The CR functions $P^{\tau} A_{\tau} \varphi_{j}$ form an orthonormal basis of $H^{2}\left(\partial M_{\tau}, d \mu_{\tau}\right)$.

Since $A_{\tau}=\Delta^{\frac{m-1}{8}} \bmod \Psi^{\frac{m-1}{4}-1}$, the basis $\left\{u_{j}^{\tau}\right\}$ is almost orthonormal, i.e. the Gram matrix $\left(\left\langle u_{j}^{\tau}, u_{k}^{\tau}\right\rangle\right)=I+K_{\tau}$ where $K_{\tau}$ is a compact operator.

3.2. Pointwise Weyl laws and norms for complexifed eigenfunctions. We denote by

$$
\Pi_{I_{\lambda}}(x, y)=\sum_{j: \lambda_{j} \in I_{\lambda}} \varphi_{j}(x) \varphi_{j}(y)
$$

the spectral projections for $\sqrt{\Delta}$ corresponding to the interval $I_{\lambda}$, which we take to be either $[0, \lambda]$ or $[\lambda, \lambda+1]$. We analytically continue the spectral projection kernels and restrict to the anti-diagonal to obtain the pluri-subharmonic kernels

$$
\Pi_{I_{\lambda}}^{\mathbb{C}}(\zeta, \bar{\zeta})=\sum_{j: \lambda_{j} \in I_{\lambda}}\left|\varphi_{\lambda_{j}}^{\mathbb{C}}(\zeta)\right|^{2}
$$

Since they are of exponential growth, we also define their 'tempered' analogues,

$$
P_{I_{\lambda}}^{\tau}(\zeta, \bar{\zeta})=\sum_{j: \lambda_{j} \in I_{\lambda}} e^{-2 \tau \lambda_{j}}\left|\varphi_{\lambda_{j}}^{\mathbb{C}}(\zeta)\right|^{2}, \quad(\sqrt{\rho}(\zeta) \leq \tau) .
$$

In $[\mathbf{Z 2}]$ we give a pointwise local Weyl law and remainder estimate for complexified spectral projections:

Proposition 3.8. [Z2] On any compact real analytic Riemannian manifold $(M, g)$ of dimension $m$, we have

$$
P_{[0, \lambda]}(\zeta, \bar{\zeta})=(2 \pi)^{-m}\left(\frac{\lambda}{\sqrt{\rho(\zeta)}}\right)^{\frac{m-1}{2}}\left(\frac{\lambda}{(m-1) / 2+1}+O(1)\right) ;
$$

with remainders uniform in $\zeta$.

The pointwise Weyl laws imply upper bounds on sup norms of complexified eigenfunctions (less precise bounds are proved in [Bou, GLS].) 
Proposition 3.9. $[\mathbf{Z 2}]$ Suppose $(M, g)$ is real analytic of dimension $m$. Then,

1) For $\tau \geq \frac{C}{\lambda}$ and $\sqrt{\rho}(\zeta)=\tau$, there exists $C_{g}>0$ (depending only on the metric) so that

$$
C_{g} \lambda_{j}^{-\frac{m-1}{2}} e^{\tau \lambda} \leq \sup _{\zeta \in M_{\tau}}\left|\varphi_{\lambda}^{\mathbb{C}}(\zeta)\right| \leq C \lambda^{\frac{m-1}{4}+\frac{1}{2}} e^{\tau \lambda} .
$$

By Corollary 3.7 the operator $P^{\tau} A^{\tau}$ is unitary so that $\left\{P^{\tau} A_{\tau} \varphi_{j}\right\}$ are orthonormal. They are asymptotically the same as

$$
\lambda_{j}^{\frac{m-1}{4}} P^{\tau} \varphi_{j}=\left.\lambda_{j}^{\frac{m-1}{4}} e^{-\tau \lambda_{j}} \varphi_{j}^{\mathbb{C}}\right|_{\partial M_{\tau}},
$$

hence these are almost normalized to have $L^{2}$-norms equal to one.

\section{The wave group in the complex domain}

To prove Proposition 2, we need to consider the symmetries of the matrix elements (or Wigner measures) in Definition 3. The key operator in studying restriction to complexified geodesics is the composition

$$
\gamma_{x, \xi}^{\tau *} \circ P^{\tau}: L^{2}(M) \rightarrow \mathcal{O}^{0}\left(\partial S_{\tau}\right)
$$

where $\gamma_{x, \xi}^{\tau *}$ is the pullback under (5). To prove Proposition 2, we show that to leading order, this operator intertwines the wave group $U^{t}=$ $e^{i t \sqrt{\Delta}}$ on $M$ with the translation operator $T_{t} f(s+i \tau)=f(s+t+i \tau)$ on $\partial S_{\tau}$. On the infinitesimal level, $\gamma_{x, \xi}^{\tau *} \circ P^{\tau}$ intertwines $\frac{\partial}{\partial t}$ with the generator $\Xi_{\sqrt{H}}$ of the geodesic flow. Here and below, we identify a vector field $\Xi$ with the differential operator $\Xi(f)=d f(\Xi)$.

It is simpler to work with a unitarily equivalent "wave group" $\tilde{V}_{\tau}^{t}$ acting on the $\mathcal{O}^{0}\left(\partial M_{\tau}\right)$. In this section, we define and study this wave group. The symbol calculus of Toeplitz Fourier integral operators shows that it is essentially the compression to $\mathcal{O}^{0}\left(\partial M_{\tau}\right)$ of translation by the (non-holomorphic) Hamilton flow of $\sqrt{\rho}$. This makes it evident that pullback by $\gamma_{x, \xi}^{\tau}$ intertwines the wave group with translations.

\subsection{The wave group $\tilde{V}_{\tau}^{t}$.}

We first define the operators $V_{\tau}^{t}$ :

Definition 4.1. Let $A_{\tau}=\left(P^{\tau *} P^{\tau}\right)^{-\frac{1}{2}}$ (43). Then set

$$
V_{\tau}^{t}=P^{\tau} U^{t} A_{\tau}^{2} P^{\tau *}: \mathcal{O}^{0}\left(\partial M_{\tau}\right) \rightarrow \mathcal{O}^{0}\left(\partial M_{\tau}\right) \text {. }
$$

The advantage of $V_{\tau}^{t}$ is that its eigenfunctions are natural:

$$
V_{\tau}^{t} P^{\tau} \varphi_{j}=e^{i t \lambda_{j}} P^{\tau} \varphi_{j}
$$

Indeed, from Proposition $3.6, A_{\tau}^{2} P^{\tau *}$ is a left inverse to $P^{\tau}$. Hence it suffices to observe that

$$
P^{\tau} U^{t} A_{\tau}^{2} P^{\tau *} P^{\tau} \varphi_{j}=e^{i t \lambda_{j}} P^{\tau} \varphi_{j}
$$


The disadvantage is that $V_{\tau}^{t}$ is not a unitary group and is not even normal since the $A_{\tau}^{2}$ factors moves to the left side of $U^{t}$ when taking the adjoint and in general $U^{t}$ and $A_{\tau}$ do not commute. So the spectral theorem does not apply to $V_{\tau}^{t}$. This defect is remedied by:

Definition 4.2. Define

$$
\tilde{V}_{\tau}^{t}=P^{\tau} A_{\tau} U^{t} A_{\tau} P^{\tau *}: \mathcal{O}^{0}\left(\partial M_{\tau}\right) \rightarrow \mathcal{O}^{0}\left(\partial M_{\tau}\right) .
$$

Proposition 4.3. $\tilde{V}_{\tau}^{t}$ is a unitary group with eigenfunctions

$$
\tilde{V}_{\tau}^{t} P^{\tau} A_{\tau} \varphi_{j}=e^{i t \lambda_{j}} P^{\tau} A_{\tau} \varphi_{j} .
$$

Proof. By Proposition 3.6,

$$
\begin{aligned}
\tilde{V}_{\tau}^{t} \tilde{V}_{\tau}^{t *}= & P^{\tau} A_{\tau} U^{t} A_{\tau} P^{\tau *} P^{\tau} A_{\tau} U^{-t} A_{\tau} P^{\tau *} \\
& =P^{\tau} A_{\tau}^{*} A_{\tau} P^{\tau *}=\Pi_{\tau}: L^{2}\left(\partial M_{\tau}\right) \rightarrow L^{2}\left(\partial M_{\tau}\right),
\end{aligned}
$$

so that $\tilde{V}_{\tau}^{t}$ is unitary. Also,

$$
\begin{aligned}
\tilde{V}_{\tau}^{t} \tilde{V}_{\tau}^{s}= & P^{\tau} A_{\tau} U^{t} A_{\tau} P^{\tau *} P^{\tau} A_{\tau} U^{s} A_{\tau} P^{\tau *} \\
& =P^{\tau} A_{\tau} U^{t+s} A_{\tau} P^{\tau *}=\tilde{V}_{\tau}^{t+s}: L^{2}\left(\partial M_{\tau}\right) \rightarrow L^{2}\left(\partial M_{\tau}\right) .
\end{aligned}
$$

Similarly,

$$
\tilde{V}_{\tau}^{t} P^{\tau} A_{\tau} \varphi_{j}=P^{\tau} A_{\tau} U^{t} A_{\tau} P^{\tau *} P^{\tau} A_{\tau} \varphi_{j}=e^{i t \lambda_{j}} P^{\tau} A_{\tau} \varphi_{j} .
$$

q.e.d.

4.2. $V_{\tau}^{t}$ as a Fourier integral Toeplitz operator. The following Proposition states the analogue of Propositions 3.1-3.3 for $V_{\tau}^{t}$. It shows that $V_{\tau}^{t}$ is a Fourier integral operator with complex phase of Hermite type on $\mathcal{O}^{0}\left(\partial M_{\tau}\right) \subset L^{2}\left(\partial M_{\tau}\right)$ which is "adapted" to the graph of the Hamiltonian flow of $\sqrt{\rho}$ on the symplectic cone $\Sigma_{\tau}$ associated to the Hardy space $\mathcal{O}^{0}\left(\partial M_{\tau}\right)$ in the sense of [BoGu] (see $\left.\S 2\right)$.

Proposition 4.4. $V_{\tau}^{t}$ and $\tilde{V}_{\tau}^{t}$ are Fourier integral operators with complex phase of Hermite type on $\mathcal{O}^{0}\left(\partial M_{\tau}\right) \subset L^{2}\left(\partial M_{\tau}\right)$ adapted to the graph of the Hamiltonian flow of $\sqrt{\rho}$ on $\Sigma_{\tau}$. They have the same canonical relations and principal symbols

$$
\sigma_{V_{\tau}^{t}}=|\xi|^{\frac{m-1}{4}} \sigma_{P^{\tau}} \circ \sigma_{U^{t}} \circ \sigma_{P^{\tau *}} .
$$

Proof. That both operators have the same canonical relation and symbol is obvious because they only differ in the order of the pseudodifferential operator $A_{\tau}$, which has a scalar symbol. By definition and by Proposition 3.1, $\tilde{V}_{\tau}^{t}$ is a composition of Fourier integral operators with complex phase, and all are associated to canonical graphs and equivalence relations. Moreover all are operators of Hermite type. If follows that the composition is transversal, so that $\tilde{V}_{\tau}^{t}$ is also a Fourier integral operator with complex phase and of Hermite type. 
The underlying complex canonical transformation is

$$
\begin{aligned}
& \mathcal{G}_{\tau, t} \circ \mathcal{G}_{\tau}^{*}=\left\{\left(\zeta, p_{\zeta}, \zeta^{\prime}, p_{\zeta^{\prime}}\right\} \subset T^{*} \widetilde{\partial M}_{\tau} \times T^{*} \widetilde{\partial M}_{\tau}:\right. \\
& \left.\exists(y, \eta) \in T^{*} M:\left(\zeta, p_{\zeta}\right) \sim G^{i \tau+t}(y, \eta), \quad\left(\zeta^{\prime}, p_{\zeta^{\prime}}\right) \sim G^{i \tau}(y, \eta)\right\}
\end{aligned}
$$

where $\mathcal{G}_{\tau, t}$ is defined in (35). Thus

$$
\left(\zeta, p_{\zeta}\right) \sim G^{t}\left(\zeta^{\prime}, p_{\zeta}^{\prime}\right)
$$

and there is the additional constraint that $\left(\zeta^{\prime}, p_{\zeta^{\prime}}\right) \sim G^{i \tau}(y, \eta) \in \Sigma_{\tau}$.

The real point set of this canonical relation is its intersection with $\Sigma_{\tau} \times \Sigma_{\tau}$, and then we have

$$
\mathcal{G}_{\tau, t} \circ \mathcal{G}_{\tau}^{*} \cap \Sigma_{\tau} \times \Sigma_{\tau}=\left\{\left(\zeta, p_{\zeta}, \zeta^{\prime}, p_{\zeta^{\prime}}\right\} \subset \Sigma_{\tau} \times \Sigma_{\tau}:\left(\zeta, p_{\zeta}\right)=G^{t}\left(\zeta^{\prime}, p_{\zeta^{\prime}}\right)\right\} .
$$

But then $\zeta^{\prime} \in \partial M_{\tau}, p_{\zeta}=d^{c} \sqrt{\rho}$ and by Proposition 1.5 and Lemma 3.4,

$$
G^{t}\left(\zeta^{\prime}, d^{c} \sqrt{\rho}\right)=\left(\exp t \Xi_{\sqrt{\rho}}\left(\zeta^{\prime}\right), d^{c} \sqrt{\rho}\right) .
$$

It follows that $V_{\tau}^{t}$ is adapted to the graph of $E G^{t} E^{-1}=\exp t \Xi_{\sqrt{\rho}}$ on $\Sigma_{\tau}$.

The symbol calculation is then a routine use of the composition theory of Fourier integral operators and therefore we only outline it here: The symbol of $U^{t}$ is well-known to be the canonical volume half-density on the graph of $G^{t}$. On the real points of the canonical relation $C_{\tau}$ of $\Pi_{\tau}$, this volume half-density is conjugated to the canonical volume halfdensity on the graph of $\exp t \Xi_{\rho}$ in $\Sigma_{\tau} \times \Sigma_{\tau}$.

q.e.d.

4.3. $V_{\tau}^{t}$ as a Toeplitz Fourier integral operator. We recall that in $[\mathbf{B o G u}]$ a special symbol calculus was developed for Fourier integral operators of Hermite type adapted to symplectic diffeomorphisms, i.e. for compressions (restrictions) $\Pi_{\tau} F \Pi_{\tau}$ of Fourier integral operators $F$ of complex type to the Hardy space $H^{2}\left(\partial M_{\tau}\right.$. Since the operators $\tilde{V}_{\tau}^{t}$ and $V_{\tau}^{t}$ of this type, they have Toeplitz symbols in this sense. In the next Proposition, we calculate these symbols and put the operators into a more geometric form of Toeplitz quantizations of a Hamiltonian flow. Such dynamical Toeplitz quantizations were studied by Boutet de Monvel-Guillemin [BoGu] and in [Z3].

To state the next Proposition, we need some further notation and background on symbols of Szegö projectors. We recall from $[\mathbf{B o G u}]$ that $\sigma_{\Pi_{\tau}}$ is a rank one projector onto a ground state $e_{\Lambda}$ in the quantization of the symplectic transversal $T \Sigma^{\perp} \subset T^{*} \partial M_{\tau}$ to $T \Sigma$. The ground state is annihilated by a Lagrangian system of Cauchy-Riemann equations corresponding to a Lagrangian subspace $\Lambda \subset T \Sigma$.

The time evolution of $\Pi_{\tau}$ under the flow $g_{\tau}^{t}$ is defined by

$$
\Pi_{\tau}^{t}=g_{\tau}^{-t} \Pi_{\tau} g_{\tau}^{t} .
$$


It is another Szegö projector adapted to the symplectic cone $\Sigma_{\tau}$; since $g^{t}$ is not a family of holomorphic maps in general, $\Pi_{\tau}^{t}$ is associated to a new CR (complex) structure and translation by $g_{\tau}^{t}$ does not commute with $\Pi_{\tau}$. But $\Sigma_{\tau}$ is invariant under the flow and $g^{t}$ clearly commutes with the identity map on $\Sigma_{\tau}$. The change in the range of $\Pi_{\tau}^{t}$ under $t$ is encoded to leading order in the change of the principal symbol.

Under $d g_{\tau}^{t}$, the Lagrangian $\Lambda_{\tau}$ goes to a new Lagrangian $\Lambda_{\tau}^{t}$ and $\sigma_{\Pi_{\tau}^{t}}$ is a rank one projector onto a ground state $e_{\Lambda_{\tau}^{t}}$ depending on $t$. If we right compose by $\Pi_{\tau}$ the composite symbol is

$$
\sigma\left(\Pi_{\tau} \Pi_{\tau}^{t} \Pi_{\tau}\right)=\left|\left\langle e_{\Lambda_{\tau}}, e_{\Lambda_{\tau}^{t}}\right\rangle\right|^{2} \sigma_{\Pi_{\tau}} .
$$

The overlap $\left\langle e_{\Lambda_{\tau}}, e_{\Lambda_{\tau}^{t}}\right\rangle$ is an inner product of two Gaussians and is calculated explicitly in $[\mathbf{Z 3}]$. It is nowhere vanishing. Define

$$
\sigma_{\tau, t}=\left\langle e_{\Lambda_{\tau}^{t}}, e_{\Lambda_{\tau}}\right\rangle^{-1}
$$

Proposition 4.5. Let $g_{\tau}^{t}=\exp t \Xi_{\sqrt{\rho}}$ on $\partial M_{\tau}$. Then there exists a pseudo-differential operator $\hat{\sigma}_{\tau, t}$ with principal symbol given by $\sigma_{\tau, t}$ times a phase $e^{i \theta_{t, \tau}}$ so that

$$
V_{\tau}^{t}:=\Pi_{\tau} g_{\tau}^{t} \hat{\sigma}_{t, \tau} \Pi_{\tau}, \quad \tilde{V}_{\tau}^{t}=\Pi_{\tau} \sqrt{\hat{\sigma}_{t, \tau}} g_{\tau}^{t} \sqrt{\hat{\sigma}_{t, \tau}} \Pi_{\tau}
$$

Proof. Each side of each formula is an elliptic Toeplitz Fourier integral operator adapted to the graph of $g_{\tau}^{t}$. In the case of $V_{\tau}^{t}$ and $\tilde{V}_{\tau}^{t}$ this follows from Proposition 3.3 and by the composition theorem for such operators in $[\mathbf{B o G u}]$. In the case of $\Pi_{\tau} g_{\tau}^{t} \sigma_{t, \tau} \Pi_{\tau}$ it follows similarly from the fact that $\Pi_{\tau}$ is a Toeplitz operator and from the simple composition with pullback by $g_{\tau}^{t}$ (see $\S 2.1$ ).

We next verify that they have the same principal symbol. To compute the symbols we use Proposition 4.3. On the principal symbol level it implies that

$\sigma_{\Pi_{\tau}} \circ \sigma_{\tau,-t} \sigma_{g^{-t} \Pi_{\tau} g^{t}} \sigma_{t, \tau} \circ \sigma_{\Pi_{\tau}}=\sigma_{\Pi_{\tau}} \leftrightarrow\left|\sigma_{\tau, t}\right|^{2} \sigma_{\Pi_{\tau}} \circ \sigma_{g^{-t} \Pi_{\tau} g^{t}} \circ \sigma_{\Pi_{\tau}}=\sigma_{\Pi_{\tau}}$.

Then

$$
\sigma_{\Pi_{\tau}} \circ \sigma_{g^{-t} \Pi_{\tau} g^{t}} \circ \sigma_{\Pi_{\tau}}=\left|\left\langle e_{\Lambda_{\tau}^{t}}, e_{\Lambda_{\tau}}\right\rangle\right|^{2}
$$

It follows that

$$
\left|\sigma_{\tau, t}\right|^{2}=\left|\left\langle e_{\Lambda_{\tau}^{t}}, e_{\Lambda_{\tau}}\right\rangle\right|^{-2}
$$

Clearly the symbol (50) is a solution, and is unique up to a phase $e^{i \theta_{t, \tau}}$. Existence of $\hat{\sigma}_{\tau, t}$ then follows by an inductive argument on the symbol order.

q.e.d.

\section{Restriction to geodesics I: Intertwining relations}

The purpose of this section is to prove that the restriction $\gamma_{x, \xi}^{\tau *}$ intertwines translation on $\mathbb{R}$ and translation by the geodesic flow on $\partial M_{\tau}$. 
There are several further intertwining relations of this kind, both infinitesimal and global. The one we need for the proof of Proposition 2 is a $T^{*} T$ version on $\partial M_{\tau}$, and so it is emphasized in the following:

Proposition 5.1. On $L^{2}\left(\partial M_{\tau}\right)$, we have

$$
V_{\tau}^{* t}\left(\left(\gamma_{x, \xi}^{\tau}\right)^{*}\right)^{*} O p_{\lambda_{j}}(a)\left(\gamma_{x, \xi}^{\tau}\right)^{*} V_{\tau}^{t} \simeq \Pi_{\tau}\left(\left(\gamma_{x, \xi}^{\tau}\right)^{*}\right)^{*} T_{t}^{*} O p_{\lambda_{j}}(a) T_{t}\left(\gamma_{x, \xi}^{\tau}\right)^{*} \Pi_{\tau}
$$

Remark 5.2. Here, $O p_{\lambda_{j}}(a)$ is a semi-classical pseudo-differential operator $[\mathbf{Z w}]$, and $\simeq$ means that both sides belong to the same class of Fourier integral operators and have the same principal symbols. Above, $\left(\gamma_{x, \xi}\right)^{*}$ denotes the pullback under the map $\gamma_{x, \xi}$ and the additional * denotes the adjoint of this operator.

By adding lower order terms to the symbols we can arrange the left side to equal the right modulo Toeplitz smoothing operators. We begin by using Proposition 4.5 to show that Proposition 5.1 is equivalent to

Lemma 5.3. We have,

$$
\begin{aligned}
& \Pi_{\tau}\left(\left(\gamma_{x, \xi}^{\tau}\right)^{*}\right)^{*} T^{t *} O p_{\lambda}(a) T^{t}\left(\gamma_{x, \xi}^{\tau}\right)^{*} \Pi_{\tau} \\
& \simeq \Pi_{\tau} \overline{\sigma_{\tau t}} g^{-t} \Pi_{\tau}\left(\left(\gamma_{x, \xi}^{\tau}\right)^{*}\right)^{*} O p_{\lambda_{j}}(a)\left(\gamma_{x, \xi}^{\tau}\right)^{*} \Pi_{\tau} \sigma_{\tau t} g^{t} \Pi_{\tau},
\end{aligned}
$$

in the sense that the operators on each side are Hermite Fourier integral operators (Toeplitz operators) adapted to the symplectic embedding $\iota_{x, \xi}^{\tau}$ : $\mathbb{R}_{+} \gamma_{x, \xi}^{\tau} \subset \Sigma_{\tau}$ and having the same principal symbol.

Indeed the equivalence follows immediately from Proposition 4.5 and from the fact that

$$
\left(\gamma_{x, \xi}^{\tau}\right)^{*} \Pi_{\tau}: \mathcal{O}^{0}\left(\partial M_{\tau}\right) \rightarrow \mathcal{O}_{l o c}^{-1}\left(\partial S_{\tau}\right)
$$

is the adjoint of a Toeplitz Hermite Fourier integral operator adapted to the symplectic embedding $\mathbb{R}_{+} \gamma_{x, \xi}^{\tau} \subset \Sigma_{\tau}$. The latter statement is proved in Theorem 9.1 of $[\mathbf{B o G u}$. All of the hypotheses of that theorem are satisfied by the map

$$
\gamma_{x, \xi}^{\tau} \times \gamma_{x, \xi}^{\tau}: \mathbb{R} \times \mathbb{R} \rightarrow \partial M_{\tau} \times \partial M_{\tau} .
$$

It follows that

$$
\left(\gamma_{x, \xi}^{\tau}\right)^{*} \Pi_{\tau}\left(\left(\gamma_{x, \xi}^{\tau}\right)^{*}\right)^{*}: \mathcal{O}^{0}\left(\partial M_{\tau}\right) \rightarrow \mathcal{O}^{0}\left(\partial M_{\tau}\right)
$$

is a Toeplitz operator quantizing the symplectic sub-cone $\mathbb{R}_{+} \gamma_{x, \xi}^{\tau} \subset \Sigma_{\tau}$, i.e. having the real points of its canonical relation along $\Delta_{\mathbb{R}_{+}} \gamma_{x, \xi}^{\tau} \times \mathbb{R}_{+} \gamma_{x, \xi}^{\tau}$. It has a paramatrix of the form,

$$
\Pi_{\tau}\left(\gamma_{x, \xi}^{\tau}(t), \gamma_{x, \xi}^{\tau}(s)\right)=\int_{0}^{\infty} e^{i \theta \psi\left(\left(\gamma_{x, \xi}^{\tau}(t), \gamma_{x, \xi}^{\tau}(s)\right)\right.} A\left(\left(\gamma_{x, \xi}^{\tau}(t), \gamma_{x, \xi}^{\tau}(s)\right), \theta\right) d \theta,
$$

obtained by pulling back the parametrix (33). 
Furthermore, Theorem 9.1 of $[\mathbf{B o G u}]$ asserts that, for any Toeplitz operator $\Pi_{\tau} Q \Pi_{\tau}$ of order $r$,

$$
\left(\gamma_{x, \xi}^{\tau}\right)^{*} \Pi_{\tau} Q \Pi_{\tau} \text { is a Toeplitz operator of order } \mathrm{r} \text { on } \partial S_{\tau},
$$

whose principal symbol is the restriction of $\sigma_{Q}$ to $\mathbb{R}_{+} \gamma_{x, \xi}^{\tau}$.

\subsection{Proof of Lemma 5.3.}

Proof. The right side is the same as

$$
\Pi_{\tau} \overline{\sigma_{\tau t}} g^{-t} \Pi_{\tau} g^{t} g^{-t}\left(\left(\gamma_{x, \xi}^{\tau}\right)^{*}\right)^{*} O p_{\lambda_{j}}(a)\left(\gamma_{x, \xi}^{\tau}\right)^{*} g^{t} g^{-t} \Pi_{\tau} g^{t} g^{-t} \sigma_{\tau t} g^{t} \Pi_{\tau} .
$$

We use the obvious intertwining relations

$$
T^{t}\left(\gamma_{x, \xi}^{\tau}\right)^{*}=\left(\gamma_{x, \xi}^{\tau}\right)^{*} g_{\tau}^{t}, \quad\left(\left(\gamma_{x, \xi}^{\tau}\right)^{*}\right)^{*} T^{-t}=g^{-t}\left(\left(\gamma_{x, \xi}^{\tau}\right)^{*}\right)^{*}
$$

to get

$$
g^{-t}\left(\left(\gamma_{x, \xi}^{\tau}\right)^{*}\right)^{*} O p_{\lambda_{j}}(a)\left(\gamma_{x, \xi}^{\tau}\right)^{*} g^{t}=\left(\left(\gamma_{x, \xi}^{\tau}\right)^{*}\right)^{*} T_{t}^{*} O p_{\lambda_{j}}(a) T^{t}\left(\gamma_{x, \xi}^{\tau}\right)^{*},
$$

and thus it suffices to prove

$$
\begin{aligned}
& \Pi_{\tau}\left(\left(\gamma_{x, \xi}^{\tau}\right)^{*}\right)^{*} T^{t *} O p_{\lambda}(a) T^{t}\left(\gamma_{x, \xi}^{\tau}\right)^{*} \Pi_{\tau} \\
& \simeq \Pi_{\tau} \overline{\sigma_{\tau t}} g^{-t} \Pi_{\tau} g^{t}\left(\left(\gamma_{x, \xi}^{\tau}\right)^{*}\right)^{*} T_{t}^{*} O p_{\lambda_{j}}(a) T^{t}\left(\gamma_{x, \xi}^{\tau}\right)^{*} g^{-t} \Pi_{\tau} g^{t} g^{-t} \sigma_{\tau t} g^{t} \Pi_{\tau} .
\end{aligned}
$$

Now $T_{t}^{*} O p_{\lambda_{j}}(a) T^{t}$ appears on both sides of the purported formula and is a completely general pseudo-differential operator. Hence (57) is equivalent to

$$
\begin{aligned}
& \Pi_{\tau}\left(\left(\gamma_{x, \xi}^{\tau}\right)^{*}\right)^{*} O p_{\lambda}(b)\left(\gamma_{x, \xi}^{\tau}\right)^{*} \Pi_{\tau} \\
& \simeq \Pi_{\tau} \overline{\sigma_{\tau t}} g^{-t} \Pi_{\tau} g^{t}\left(\left(\gamma_{x, \xi}^{\tau}\right)^{*}\right)^{*} O p_{\lambda}(b)\left(\gamma_{x, \xi}^{\tau}\right)^{*} g^{-t} \Pi_{\tau} g^{t} g^{-t} \sigma_{\tau t} g^{t} \Pi_{\tau},
\end{aligned}
$$

for any $O p_{\lambda}(b)$.

We further observe that $g^{-t} \sigma_{\tau t} g^{t}=\sigma_{\tau t} \circ g^{-t}$. We thus need to show that

$$
\begin{aligned}
& \Pi_{\tau}\left(\left(\gamma_{x, \xi}^{\tau}\right)^{*}\right)^{*} O p_{\lambda}(b)\left(\gamma_{x, \xi}^{\tau}\right)^{*} \Pi_{\tau} \\
& \simeq \Pi_{\tau} \overline{\sigma_{\tau t}} \Pi_{\tau}^{t}\left(\left(\gamma_{x, \xi}^{\tau}\right)^{*}\right)^{*} O p_{\lambda}(b)\left(\gamma_{x, \xi}^{\tau}\right)^{*} \Pi_{\tau}^{t}\left(\sigma_{\tau t} \circ g^{-t}\right) \Pi_{\tau} .
\end{aligned}
$$

To prove this, we show that

$$
\begin{aligned}
& \Pi_{\tau} \overline{\sigma_{\tau t}} \Pi_{\tau}^{t}\left(\left(\gamma_{x, \xi}^{\tau}\right)^{*}\right)^{*} O p_{\lambda}(b)\left(\gamma_{x, \xi}^{\tau}\right)^{*} \Pi_{\tau}^{t}\left(\sigma_{\tau t} \circ g^{-t}\right) \Pi_{\tau} \\
& \simeq \Pi_{\tau} \overline{\sigma_{\tau t}} \Pi_{\tau}^{t} \Pi_{\tau}\left(\left(\gamma_{x, \xi}^{\tau}\right)^{*}\right)^{*} O p_{\lambda}(b)\left(\gamma_{x, \xi}^{\tau}\right)^{*} \Pi_{\tau} \Pi_{\tau}^{t}\left(\sigma_{\tau t} \circ g^{-t}\right) \Pi_{\tau} .
\end{aligned}
$$

Here, we inserted an extra factor of $\Pi_{\tau}$ to the right of the first $\Pi_{\tau}^{t}$ and to the left of the second. To prove (60) we use the $\square_{b}^{\tau}=\bar{\partial}_{b}^{\tau *} \bar{\partial}_{b}^{\tau}$ operator on $\partial M_{\tau}$, where $\bar{\partial}_{b}^{\tau}$ is the Cauchy-Riemann operator associated to the CR structure of $\partial M_{\tau}$ as a real hypersurface in $M_{\tau}$. Thus, $\Pi_{\tau}$ is the orthogonal projection onto the kernel of $\square_{b}^{\tau}$. Since $\partial M_{\tau}$ is strictly 
pseudo-convex, there is a spectral gap between the 0 eigenvalue of $\square_{b}^{\tau}$ and its first positive eigenvalue. Thus there exists a pseudo-differential Green's operator $G_{\tau}$ of order -2 such that

$$
\square_{b}^{\tau} G_{\tau}=I d-\Pi_{\tau} .
$$

We use this to write

$$
\Pi_{\tau} \sigma \Pi_{\tau}^{t}=\Pi_{\tau} \sigma \Pi_{\tau}^{t} \Pi_{\tau}+\Pi_{\tau} \sigma \Pi_{\tau}^{t} \square_{b}^{\tau} G_{\tau} .
$$

We then observe that the principal symbol of the zeroth order Toeplitz operator $\Pi_{\tau} \sigma \Pi_{\tau}^{t} \square_{b}^{\tau} G_{\tau}$ vanishes. Indeed, since $\Pi_{\tau} \square_{b}^{\tau}=0$ it is the same as $\Pi_{\tau}\left[\sigma \Pi_{\tau}^{t}, \square_{b}^{\tau}\right] G_{\tau}$. But the commutator $\left[\sigma \Pi_{\tau}^{t}, \square_{b}^{\tau}\right]$ is a Toeplitz operator adapted to the identity map on $\Sigma_{\tau}$ with vanishing principal symbol, since scalar functions commute with the symbol of $\Pi_{\tau}^{t}$. It follows that $\Pi_{\tau} \sigma \Pi_{\tau}^{t} \square_{b}^{\tau} G_{\tau}$ is of order -1 , and does not contribute to the principal symbol of the right side of (60), proving the claim.

Thus we reduce to proving that the symbol of right side of $(60)$ is the same as the symbol of the left side of (59). But this follows from the fact that

$$
\Pi_{\tau} \overline{\sigma_{\tau t}} \Pi_{\tau}^{t} \Pi_{\tau} \simeq \Pi_{\tau}, \quad \Pi_{\tau} \sigma_{\tau t} \circ g^{-t} \Pi_{\tau}^{t} \Pi_{\tau} \simeq \Pi_{\tau} .
$$

Indeed, the symbol of the left side of the first expression equals

$$
\overline{\sigma_{\tau t}} \sigma_{\Pi_{\tau}} \sigma_{\Pi_{\tau}^{t}} \sigma_{\Pi_{\tau}}=\overline{\sigma_{\tau t}}\left\langle e_{\Lambda_{\tau}}, e_{\Lambda_{\tau}^{t}}\right\rangle \sigma_{\Pi_{\tau}}=\sigma_{\Pi_{\tau}}
$$

since the numerical factor cancels by $(50)$. The symbol on the left side of the second is

$$
\sigma_{\tau t} \circ g^{-t}\left\langle e_{\Lambda_{\tau}}, e_{\Lambda_{\tau}^{t}}\right\rangle \sigma_{\Pi_{\tau}}=\sigma_{\Pi_{\tau}} .
$$

Indeed, $g^{-t} \Lambda_{t}=\Lambda$ so

$$
\sigma_{\tau t} \circ g^{-t}=\left\langle e_{\Lambda_{\tau}}, e_{\Lambda_{\tau}^{t}}\right\rangle^{-1}=\overline{\sigma_{\tau t}}
$$

and so the claim follows as for the first expression. This completes the proof of Lemma 5.3 and therefore of Proposition 5.1.

q.e.d.

Differentiating Lemma 5.3 in $t$ and setting $t=0$ gives the infinitesimal version:

Corollary 5.4. Let $\sigma_{0}=\left.\frac{d}{d t}\right|_{t=0}\left\langle e_{\Lambda_{\tau}^{t}}, e_{\Lambda_{\tau}}\right\rangle^{-1}$. There exists a pseudodifferential operator $R_{-1}$ of order -1 so that,

$$
\begin{aligned}
& \Pi_{\tau}\left(\left(\gamma_{x, \xi}^{\tau}\right)^{*}\right)^{*} O p_{\lambda}\left(\left[\frac{\partial}{\partial t}, a\right]\right)\left(\gamma_{x, \xi}^{\tau}\right)^{*} \Pi_{\tau} \\
& \left.\simeq \Pi_{\tau}\left[\Xi_{\sqrt{\rho}}+\sigma_{0}+R_{-1}\right),\left(\left(\gamma_{x, \xi}^{\tau}\right)^{*}\right)^{*} O p_{\lambda_{j}}(a)\left(\gamma_{x, \xi}^{\tau}\right)^{*}\right] \Pi_{\tau} .
\end{aligned}
$$

Proof.

$$
\frac{d}{d t} \Pi_{t=0} g^{t} \sigma_{t, \tau} \Pi_{\tau}=\Pi_{\tau}\left(\Xi_{\sqrt{\rho}}+\left.\frac{d}{d t}\right|_{t=0} \sigma_{t, \tau}\right) \Pi_{\tau} .
$$


By (50), the second term is

$$
\left.\frac{d}{d t}\right|_{t=0} \sigma_{\tau, t}=\left.\frac{d}{d t}\right|_{t=0}\left\langle e_{\Lambda_{\tau}^{t}}, e_{\Lambda_{\tau}}\right\rangle^{-1} .
$$

q.e.d.

Alternatively, one can construct a paramatrix for $\left(\gamma_{x, \xi}^{\tau}\right)^{*} \Pi_{\tau}$ by pulling back the parametrix (33), to get

$$
\Pi_{\tau}\left(\gamma_{x, \xi}(t), \zeta\right)=\int_{0}^{\infty} e^{i \theta \psi\left(\left(\gamma_{x, \xi}^{\tau}(t), \zeta\right)\right.} A\left(\left(\gamma_{x, \xi}^{\tau}(t), \zeta, \theta\right) d \theta\right.
$$

Applying $\frac{\partial}{\partial t}$ changes the amplitude to

$$
i \theta \frac{\partial}{\partial t} \psi\left(\left(\gamma_{x, \xi}^{\tau}(t), \zeta\right) A+\frac{\partial}{\partial t} A\left(\left(\gamma_{x, \xi}(t), \zeta, \theta\right) .\right.\right.
$$

The first term is $\partial \psi\left(\left(\gamma_{x, \xi}^{\tau}(t), \zeta\right) \cdot \gamma_{x, \xi}^{\tau^{\prime}}(t)\right.$, and on the diagonal $\partial \psi$ is the contact form, which evaluates to 1 on $\gamma_{x, \xi}^{\tau^{\prime}}(t)$. Similarly, the symbol of $\Xi_{\sqrt{\rho}}$ evaluates to $\theta$.

\section{Lebesgue limits of matrix elements: Proof of Proposition 2}

In this section, we use the intertwining relation in Proposition 5.1 to prove that matrix elements

$$
\left\langle a U_{j}^{\mathbf{I}_{\tau} x, \xi}, U_{j}^{\mathbf{I}_{\tau}, x, \xi}\right\rangle
$$

of compactly supported multiplication operators on $\partial S_{\tau}$ with respect to the $L^{2}$ normalized pullbacks of Definition 2 are asymptotically invariant under translation $T_{t}: L^{2}(\mathbb{R}) \rightarrow L^{2}(\mathbb{R})$. It is simplest to show

Proposition 6.1. Let $a \in C_{0}^{\infty}\left(I_{\tau}\right)$. Then

$$
\left\langle\frac{\partial}{\partial t} a,\left|U_{j}^{\mathbf{I}_{\tau}, x, \xi}\right|^{2}\right\rangle=o_{I_{\tau}}(1), \quad \lambda_{j} \rightarrow \infty .
$$

The invariance property scales with the normalization of the pullbacks. We therefore use the preliminary normalization

$$
u_{j}^{\tau}=\frac{\varphi_{j}^{\mathbb{C}}}{\left\|\varphi_{j}^{\mathbb{C}}\right\|_{L^{2}\left(\partial M_{\tau}\right)}}
$$

and later divide again by the mass of the pullback on $I_{\tau}$.

Proof. By differentiating Proposition 4.5 in $t$ (and setting $t=0$ ), there exists a pseudo-differential operator $\sigma_{0}$ on $\partial M_{\tau}$ of order zero so that,

$$
\Pi_{\tau}\left(\Xi_{\sqrt{\rho}}+\sigma_{0}\right) \Pi_{\tau} u_{j}^{\tau}=i \lambda_{j} u_{j}^{\tau} .
$$

We then use Corollary 5.4 to obtain, 


$$
\begin{aligned}
& \left\langle\left(\frac{\partial}{\partial t} a\right)\left(\gamma_{x, \xi}^{\tau}\right)^{*} u_{j}^{\tau},\left(\gamma_{x, \xi}^{\tau}\right)^{*} u_{j}^{\tau}\right\rangle_{\mathbb{R}} \\
= & \left\langle\left[\frac{\partial}{\partial t}, a\right]\left(\gamma_{x, \xi}^{\tau}\right)^{*} u_{j}^{\tau},\left(\gamma_{x, \xi}^{\tau}\right)^{*} u_{j}^{\tau}\right\rangle_{\mathbb{R}} \\
= & \left.\left\langle\left[\Xi_{\sqrt{\rho}}+\sigma_{0}+R_{-1}\right),\left(\left(\gamma_{x, \xi}^{\tau}\right)^{*}\right)^{*} O p_{\lambda_{j}}(a)\left(\gamma_{x, \xi}^{\tau}\right)^{*}\right] u_{j}^{\tau}, u_{j}^{\tau}\right\rangle_{M} \\
= & \left\langle\left[R_{-1},\left(\left(\gamma_{x, \xi}^{\tau}\right)^{*}\right)^{*} O p_{\lambda_{j}}(a)\left(\gamma_{x, \xi}^{\tau}\right)^{*}\right] u_{j}^{\tau}, u_{j}^{\tau}\right\rangle_{M}
\end{aligned}
$$

where $R_{-1}$ is a pseudo-differential operator of order -1 . We then apply the Cauchy-Schwartz inequality and the fact that

$$
\left\|R_{-1} u_{j}^{\tau}\right\|_{L^{2}\left(\partial M_{\tau}\right.} \leq \lambda_{j}^{-1}\left\|u_{j}^{\tau}\right\|_{L^{2}\left(\partial M_{\tau}\right)}
$$

to bound the final expression by

$$
\begin{aligned}
& \|\left\langle\left[R_{-1},\left(\left(\gamma_{x, \xi}^{\tau}\right)^{*}\right)^{*} O p_{\lambda_{j}}(a)\left(\gamma_{x, \xi}^{\tau}\right)^{*}\right] u_{j}^{\tau}, u_{j}^{\tau}\right\rangle_{M} \mid \\
\leq & \lambda_{j}^{-1}\left\|\Pi_{\tau}\left(\gamma_{x, \xi}^{\tau}\right)^{*} a_{0}\left(\gamma_{x, \xi}^{\tau}\right)^{*} \Pi_{\tau} u_{j}^{\tau}\right\|_{L^{2}\left(\partial M_{\tau}\right)} \\
\leq & \lambda_{j}^{-1}\left\|\left(\gamma_{x, \xi}^{\tau}\right)^{*} u_{j}^{\tau}\right\|_{L^{2}(\mathbf{I})} .
\end{aligned}
$$

Here, supp $a \subset \mathbf{I}$. If we divide $\gamma_{x, \xi}^{\tau *} u_{j}^{\tau}$ by $\left\|\left(\gamma_{x, \xi}^{\tau}\right)^{*} u_{j}^{\tau}\right\|_{L^{2}(I)}$ to obtain $U_{j}^{\mathbf{I}, x, \xi}$ then the latter expression tends to zero as $\lambda_{j} \rightarrow \infty$ and the sequence $\left|U_{j}^{\mathbf{I}, x, \xi}\right|^{2}$ is a sequence of probability measures on $\mathbf{I}$ whose weak* limits must be probability measures on $\mathbf{I}$ given by constant multiples of $d t$. Of course, there is only one such probability measure.

q.e.d.

Remark 6.2. If we chose to divide by the $L^{2}$ norm of $\gamma_{x, \xi}^{\tau *} u_{j}^{\tau}$ on a larger interval $J, \mathbf{I} \subset J$, then we would end up with the ratio

$$
\frac{\left\|\left(\gamma_{x, \xi}^{\tau}\right)^{*} u_{j}^{\tau}\right\|_{L^{2}(\mathbf{I})}}{\left\|\left(\gamma_{x, \xi}^{\tau}\right)^{*} u_{j}^{\tau}\right\|_{L^{2}(J)}}<1
$$

and the same estimate holds.

We give a second proof using the global propagator. Although it is essentially the same, it is worth recording because the interval on which the remainder estimate is made gets shifted by $t$ units. We begin with a pointwise Weyl law giving almost uniform bounds for restrictions of 'most' normalized complexified eigenfunctions. We recall that the lower counting (or natural) density of a subset $S \subset \mathbb{Z}_{+}$is defined by

$$
D_{*}(S)=\liminf _{N \rightarrow \infty} \frac{1}{N} \#\{j \in S: j \leq N\} .
$$


Lemma 6.3. For all compact intervals $I$ and $\epsilon>0$ there exists a subsequence $\mathcal{S}_{I, \epsilon}$ of lower counting density $D_{*}\left(\mathcal{S}_{I, \epsilon}\right) \geq 1-\epsilon$ so that

$$
\limsup _{j \rightarrow \infty, j \in \mathcal{S}_{I, \epsilon}} e^{-2 \tau \lambda_{j}} \int_{I}\left|\varphi_{\lambda_{j}}^{\mathbb{C}}\left(\gamma_{x, \xi}(t+i \tau)\right)\right|^{2} d t \leq \epsilon^{-1}|I| .
$$

Here, as above, $|I|$ is the Lebesgue measure of $I$. We set $\sqrt{\rho}=\tau$ and define

$$
M(\lambda)=(2 \pi)^{-m} \tau^{-\frac{m-1}{2}} \frac{\lambda^{\frac{m+1}{2}}}{(m-1) / 2+1} .
$$

Proof. It follows from Proposition 3.8 that for all $t \in \mathbb{R}$,

$$
\frac{1}{M(\lambda)} \sum_{j: \lambda_{j} \in I_{\lambda}} e^{-2 \tau \lambda_{j}}\left|\varphi_{\lambda_{j}}^{\mathbb{C}}\left(\gamma_{x, \xi}(t+i \tau)\right)\right|^{2}=1+O\left(\lambda^{-1}\right),
$$

and by integrating over a compact interval $I \subset \mathbb{R}$ we have

$$
\frac{1}{M(\lambda)} \sum_{j: \lambda_{j} \in I_{\lambda}} e^{-2 \tau \lambda_{j}} \int_{I}\left|\varphi_{\lambda_{j}}^{\mathbb{C}}\left(\gamma_{x, \xi}(t+i \tau)\right)\right|^{2} d t=|I|+O\left(\lambda^{-1}\right),
$$

where the remainders are uniform in $t$ resp. $I$. We then apply a simple Chebyshev inequality to (65). Let $\{X(j)\}_{j=1}^{\infty}$ be a sequence of positive real numbers satisfying

$$
M\{X(j)\}:=\lim _{N \rightarrow \infty} \frac{1}{N} \sum_{j=1}^{N} X(j)=M .
$$

Then for any $\delta>0$ there exists $N_{\delta}<\infty$ so that

$$
M-\delta \leq \frac{1}{N} \sum_{j=1}^{N} X(j) \leq M+\delta, \quad N \geq N_{\delta}
$$

For $S \subset\{1, \ldots, N\}$, let $\mathbf{P}_{N}(S)=\frac{1}{N} \# S \cap\{1, \ldots, N\}$. Then for $N \geq N_{\delta}$, and for $k \in \mathbb{Z}_{+}$,

$$
\mathbf{P}_{N}\{j \in\{1, \ldots, N\}: X(j) \leq k M\} \geq 1-\frac{M+\delta}{k M} .
$$

Setting

$$
X(j)=e^{-2 \tau \lambda_{j}}\left|\varphi_{\lambda_{j}}^{\mathbb{C}}\left(\gamma_{x, \xi}(t+i \tau)\right)\right|^{2}, k=\left[\frac{1}{\epsilon}\right]
$$

and taking $\lim \inf _{N}$ proves the Lemma.

q.e.d.

Lemma 6.3 implies Proposition 6.1 as follows: 
Proof. We assume that $a \in C_{c}^{\infty}(\mathbb{R})$. By Lemma 5.3 , we have (66)

$$
\begin{aligned}
& \left\langle a\left(\gamma_{x, \xi}^{\tau}\right)^{*} u_{j}^{\tau},\left(\gamma_{x, \xi}^{\tau}\right)^{*} u_{j}^{\tau}\right\rangle_{\mathbb{R}}=\left\langle a\left(\gamma_{x, \xi}^{\tau}\right)^{*} V_{\tau}^{t} u_{j}^{\tau},\left(\gamma_{x, \xi}^{\tau}\right)^{*} V_{\tau}^{t} u_{j}^{\tau}\right\rangle_{\mathbb{R}} \\
& =\left\langle V_{\tau}^{* t}\left(\left(\gamma_{x, \xi}^{\tau}\right)^{*}\right)^{*} a\left(\gamma_{x, \xi}^{\tau}\right)^{*} V_{\tau}^{t} u_{j}^{\tau} \cdot u_{j}^{\tau}\right\rangle_{\partial M_{\tau}} \\
& \left\langle\left(\left(\gamma_{x, \xi}^{\tau}\right)^{*}\right)^{*} T_{t}^{*} a T_{t}\left(\gamma_{x, \xi}^{\tau}\right)^{*} u_{j}^{\tau}, u_{j}^{\tau}\right\rangle_{\partial M_{\tau}}+O\left(\left\|T_{t}(a)\left(\gamma_{x, \xi}^{\tau}\right)^{*} R_{-1}\left(\lambda_{j}\right) u_{j}^{\tau}\right\|_{\partial M_{\tau}}^{2}\right) \\
& =\left\langle\left(\left(\gamma_{x, \xi}^{\tau}\right)^{*}\right)^{*}\left(T_{t} a\right)\left(\gamma_{x, \xi}^{\tau}\right)^{*} u_{j}^{\tau}, u_{j}^{\tau}\right\rangle_{\partial M_{\tau}}+O\left(\left\|\left(T_{t} a\right)\left(\gamma_{x, \xi}^{\tau}\right)^{*} R_{-1}\left(\lambda_{j}\right) u_{j}^{\tau}\right\|_{\partial M_{\tau}}^{2}\right) \\
& =\left\langle\left(\left(T_{t} a\right)\left(\gamma_{x, \xi}^{\tau}\right)^{*} u_{j}^{\tau},\left(\gamma_{x, \xi}^{\tau}\right)^{*} u_{j}^{\tau}\right\rangle_{\mathbb{R}}\right. \\
& +\lambda_{j}^{-1} O\left(\left\|\left(\gamma_{x, \xi}^{\tau}\right)^{*} u_{j}^{\tau}\right\|_{T_{t}\left(\mathbf{I}_{\tau}\right)}\right), \quad\left(\text { as } \lambda_{j} \rightarrow \infty\right)
\end{aligned}
$$

where (as above) $R_{-1}$ is a pseudo-differential operator of order -1 and $T_{t} a(s)=a(s+t)$.

We then divide by $\left(\left\|\left(\gamma_{x, \xi}^{\tau}\right)^{*} u_{j}^{\tau}\right\|_{\mathbf{I}_{\tau}}\right.$, and observe that we get translation invariance of weak* limits on the longest interval with the property that

$$
\left\{\frac{\left\|\left(\gamma_{x, \xi}^{\tau}\right)^{*} u_{j}^{\tau}\right\|_{T_{t}\left(\mathbf{I}_{\tau}\right)}}{\left\|\left(\gamma_{x, \xi}^{\tau}\right)^{*} u_{j}^{\tau}\right\|_{\mathbf{I}_{\tau}}}\right\}_{j=1}^{\infty}
$$

is bounded, or more generally is $o\left(\lambda_{j}\right)$, for the given subsequence. Lemma 6.3 implies that this happens for a subsequence of eigenvalues of full density. This concludes the proof of Proposition 2.

q.e.d.

6.1. Lebesgue limits for the family of translates. When dealing with non-periodic geodesics it is useful to consider the family

$$
\gamma_{G^{s}(x, \xi)}^{*} \varphi_{j}, \quad(s \in \mathbb{R})
$$

of pullbacks as $s, \lambda_{j}$ vary. We have,

$$
\gamma_{G^{s}(x, \xi)}^{*} \varphi_{j}(t)=\gamma_{x, \xi}^{*} \varphi_{j}(t-s),
$$

so we are considering the family of translates

$$
\mathcal{F}=\left\{T_{s} \gamma_{x, \xi}^{*} \varphi_{j}, \quad j=1,2, \ldots, s \in \mathbb{R}\right\} .
$$

The family of translates for fixed $j$ is of course not compact in $C_{b}(\mathbb{R})$ for general $\gamma_{x, \xi}$. On the other hand, for fixed $j$ the family of translates $\left\{\varphi_{j}\left(G^{s}(x, \xi)\right), \quad s \in \mathbb{R}\right\}$ is compact in $C\left(S^{*} M\right)$.

We modify Definition 2 as follows:

Definition 6.4. Let $\left\{N_{j}\right\} \subset \mathbb{R}$ and define

$$
Y_{j}^{\tau, T, x, \xi}:=U_{j}^{\mathbf{I}_{\tau, T}, G^{N_{j}}(x, \xi)}, \quad \mathbf{I}_{\tau, T}=\partial S_{\tau, T},
$$

where $\partial S_{\tau, T}:=\{t+i \tau:|t| \leq T\}$. 
We then modify the proof of Proposition 2 to show that the weak* limits of

$$
\left|Y_{j}^{\tau, T, x, \xi}\right|^{2} d t \quad \text { on the line segments } \partial S_{\tau, T}
$$

tend to normalized Lebesgue measure:

Proposition 6.5. (Lebesgue limits for moving pullbacks) Let $(x, \xi) \in$ $B_{\epsilon}^{*} M$ be any point. Then as long as $\gamma_{x, \xi}^{*} \varphi_{j} \neq 0$ (identically), the sequence $\left\{\left|Y_{j}^{\tau, T, x, \xi}\right|^{2}\right\}$ is QUE on $\mathbb{R}$ with limit measure given by normalized Lebesgue measure on each segment $\partial S_{\tau, T}$

Proof. The proof is similar to that of Proposition 2, so we only indicate how to modify it. As in the proof of Proposition 2, the key step is to generalize the intertwining relation in Proposition 5.1 to prove that matrix elements

$$
\left\langle O p_{\lambda_{j}}(a) Y_{j}^{\tau, T, x, \xi}, Y_{j}^{\tau, T, x, \xi}\right\rangle
$$

are asymptotically invariant under translation $T_{t}: L^{2}(\mathbb{R}) \rightarrow L^{2}(\mathbb{R})$. We need to replace $\gamma_{x, \xi}^{\tau *}$ by $\gamma_{G^{N_{j}(x, \xi)}}^{\tau^{*}}$ everywhere in the proof of (66). To do this, we observe that the intertwining relation generalizes to the two-parameter equivalence

$$
\begin{aligned}
& \Pi_{\tau}\left(\left(\gamma_{G^{s}(x, \xi)}^{\tau}\right)^{*}\right)^{*} T^{t *} O p_{\lambda}(a) T^{t}\left(\gamma_{G^{s}((x, \xi)}^{\tau}\right)^{*} \Pi_{\tau} \\
& \simeq \Pi_{\tau} \overline{\sigma_{\tau t}} g^{-t} \Pi_{\tau}\left(\gamma_{G^{s}((x, \xi)}^{\tau}\right)^{* *} O p_{\lambda_{j}}(a)\left(\gamma_{G^{s}(x, \xi)}^{\tau}\right)^{*} \Pi_{\tau} \sigma_{\tau t} g^{t} \Pi_{\tau},
\end{aligned}
$$

where the remainders are uniform in $s$. To see that the implicit remainders in the $\simeq$ relation are uniform in $s$ we observe that the intertwining relation holds for all $(x, \xi) \in S^{*} M$, and (since $S^{*} M$ is compact) with uniform remainders in $(x, \xi)$. Since are only specializing the estimate to points on $\gamma_{x, \xi}$, the remainders are uniform in $s$.

We then set $s=N_{j}$ and follow the steps in (68). The remainder in the last line of (68) gets replaced by the same expression with $\gamma_{G^{N_{j}}(x, \xi)}^{*} u_{j}^{\tau}$ replacing $\gamma_{x, \xi}^{*} u_{j}^{\tau}$. Due to the uniformity of the remainders in the intertwining relation (69), the remainder in the modified (68) is still of order $O\left(\lambda_{j}^{-1}\right)$ after we divide by $\left\|\gamma_{G^{N_{j}}(x, \xi)}^{*} u_{j}^{\tau}\right\|$, or equivalently, when the matrix elements are taken with respect to the $L^{2}$-normalized $Y_{j}^{\tau, T, x, \xi}$.

\section{Growth rates of restricted eigenfunctions: Proof of Lemma 1}

We apply a general compactness theorem for subharmonic functions (see [Ho, Theorem 4.1.9]): 
Let $\left\{v_{j}\right\} \subset S H(X)$ be a sequence of subharmonic functions in an open set $X \subset \mathbb{R}^{m}$ which have a uniform upper bound on any compact set. Then either $v_{j} \rightarrow-\infty$ uniformly on every compact set, or else there exists a subsequence $v_{j_{k}}$ which converges in $L_{l o c}^{1}(X)$ to some $v \in$ $L_{\text {loc }}^{1}(X) \cap S H(X)$. Further, we have ('Hartogs' Lemma):

(i) $\quad \limsup _{j} v_{j}(x) \leq v(x)$ with equality almost everywhere

(ii) For every compact subset $K \subset X$ and every continuous

function $f, \quad \lim \sup _{j \rightarrow \infty} \sup _{K}\left(v_{j}-f\right) \leq \sup _{K}(v-f)$.

(iii) In particular, if $f \geq v$; and $\epsilon>0$, then $v_{j} \leq f+\epsilon$ on $K$

for $j$ large enough.

In $[\mathbf{Z}]$ we applied this compactness theorem to prove that for the full sequence of ergodic eigenfunctions,

$$
v_{j}=\frac{1}{\lambda_{j}} \log \left|\varphi_{j}^{\mathbb{C}}(\zeta)\right|^{2} \rightarrow 2 \sqrt{\rho} \text { in } L^{1}\left(M_{\epsilon}\right) .
$$

We note that when $v_{j_{k}} \rightarrow v$ in $L_{l o c}^{1}$ then $\lim \sup _{k \rightarrow \infty} v_{j_{k}}$ need not be upper semi-continuous. If we denote by $v^{*}$ its upper semi-continuous regularization then $v=v^{*}$ almost everywhere and by the compactness theorem $\lim _{\sup _{k}} v_{j_{k}}=v$ almost everywhere.

Proof. To prove Lemma 1, we first observe that $v_{j}$ is $\mathrm{SH}$ (subharmonic) on $S_{\epsilon}$, and apply the above theorem to $\left\{v_{j}\right\}$ with $X=S_{\epsilon_{0}}$. Exactly as in $[\mathbf{Z}]$, it follows from Proposition 3.9 that the sequence $\left\{v_{j}\right\}$ is uniformly bounded above by $2|\tau|$ globally on $M_{\tau}$. Under the condition of non-uniform convergence to $-\infty$, there must exist a subsequence, which we continue to denote by $\left\{v_{j_{k}}\right\}$, which converges in $L_{l o c}^{1}\left(S_{\epsilon_{0}}\right)$ to some $v \in L_{l o c}^{1}\left(S_{\epsilon_{0}}\right) \cap S H\left(S_{\epsilon_{0}}\right)$.

Thus, $v \leq 2|\tau|$. Assume for purposes of contradiction that $v<2|\tau|-\epsilon$ on an open set $W_{\epsilon} \subset S_{\epsilon}$. Let $I_{\tau}^{\epsilon}=W_{\epsilon} \cap \partial S_{\tau}$. By Lemma 2, one has

$\left|U_{j_{k}}^{x, \xi, I_{\tau}}\right|^{2} \rightarrow \frac{1}{I_{\tau}} d t$ in the weak* topology of measures for every interval $I_{\tau}$. In particular, this holds on $I_{\tau}^{\epsilon}$. If so, we claim that $v \leq 2|\tau|-\epsilon$ on all of $\partial S_{\tau}$. If not, there is an interval $I_{\tau, \delta}$ where $v \geq 2|\tau|-\epsilon+\delta$ for some $\delta>0$. That is,

$$
\gamma_{x, \xi}^{* \tau} u_{j}^{\tau} \leq e^{(2|\tau|-\epsilon) \lambda_{j}} \text { on } I_{\tau}^{\epsilon} \text {, and } v \geq 2|\tau|-\epsilon+\delta \text { on } I_{\tau, \delta} .
$$

For any interval $I$,

$$
\frac{1}{\lambda_{j_{k}}} \log \int_{I}\left|u_{j_{k}}^{\tau}\right|^{2} \frac{d t}{|I|} \geq 2 \int_{I} v_{j_{k}} \frac{d t}{|I|} \rightarrow 2 \int_{I} v \frac{d t}{|I|} .
$$


So if $I=I_{\tau, \delta}$ we have

$$
\frac{1}{\lambda_{j_{k}}} \log \int_{I_{\tau, \delta}}\left|u_{j_{k}}^{\tau}\right|^{2} \frac{d t}{\left|I_{\tau, \delta}\right|} \geq 2(|\tau|-\epsilon+\delta / 2)
$$

for $k \geq k_{0}(\delta)$. That is,

$$
\int_{I_{\tau, \delta}}\left|u_{j_{k}}^{\tau}\right|^{2} \frac{d t}{\left|I_{\tau, \delta}\right|} \geq e^{2 \lambda_{j_{k}}(|\tau|-\epsilon+\delta / 2)} .
$$

Again we have $\left|U_{j_{k}}^{x, \xi, I_{\tau, \delta}}\right|^{2} \rightarrow \frac{1}{I_{\tau, \delta}} d t$. Now choose $T$ large enough so that both $I_{\tau}^{\epsilon}$ and $I_{\tau, \delta}$ are continued in $I_{T}=[-T, T]$. Then we also have

$$
\left|U_{j_{k}}^{x, \xi, I_{T}}\right|^{2} d t \rightarrow d t, \text { weak } * \text { on }[-T, T] .
$$

Since $I_{\tau, \delta} \subset I_{T}$, for sufficiently large $k$,

$$
\left\|\gamma_{x, \xi}^{*} u_{j_{k}}^{\tau}\right\|_{L^{2}\left(I_{T}\right)} \geq \frac{\left|I_{\tau, \delta}\right|}{2 T}\left\|\gamma_{x, \xi}^{*} u_{j_{k}}^{\tau}\right\|_{L^{2}\left(I_{\tau, \delta}\right)} \geq \frac{\left|I_{\tau, \delta}\right|}{2 T} e^{(\tau-\epsilon+\delta / 2) \lambda_{j_{k}}} .
$$

We then compare the two statements,

$$
\left|U_{j_{k}}^{x, \xi, I_{\tau}^{\epsilon}}\right|^{2} d t \rightarrow \frac{d t}{|I|} \text { weak * on } I_{\tau}^{\epsilon}, \quad\left|U_{j_{k}}^{x, \xi, I_{T}}\right|^{2} d t \rightarrow \frac{d t}{\left|I_{T}\right|} \text { weak * on } I_{T} \text {. }
$$

The conditions overlap for $a \in C_{c}^{\infty}\left(I_{\tau}^{\epsilon}\right)$, so let us choose a test function approximating the characteristic function $\mathbf{1}_{I_{\tau}^{\epsilon}}$, and in fact we may assume $a$ equals this test function. But on $I_{\tau}^{\epsilon}$,

$$
\left|U_{j_{k}}^{x, \xi, I_{T}}\right|^{2}=\left|U_{j_{k}}^{x, \xi, I_{\tau}^{\epsilon}}\right|^{2} \frac{\left.|| \gamma_{x, \xi}^{\tau *} u_{j}^{\tau}\right|_{L^{2}\left(I_{\tau}^{\epsilon}\right)}}{\| \gamma_{x, \xi}^{\tau *} u_{j}^{\tau}||_{L^{2}\left(I_{T}\right)}} \frac{\left|I_{T}\right|}{\left|I_{\tau}^{\epsilon}\right|} \leq C_{T, \epsilon, \delta} e^{-\frac{1}{2} \delta \lambda_{j}}\left|U_{j_{k}}^{x, \xi, I_{\tau}^{\epsilon}}\right|^{2}
$$

It is thus impossible that both $\left|U_{j_{k}}^{x, \xi, I_{T}}\right|^{2}$ and $\left|U_{j_{k}}^{x, \xi, I_{\tau}^{\epsilon}}\right|^{2}$ tend to 1 weakly on $I_{\tau}^{\epsilon}$.

This contradiction shows that

$$
v<2|\tau|-\epsilon \text { on } W_{\epsilon} \Longrightarrow v<2|\tau|-\epsilon \text { on } \partial S_{\tau} .
$$

It follows that

$$
\limsup _{k \rightarrow \infty} \frac{1}{\lambda_{j_{k}}} \log \left|\gamma_{x, \xi}^{*} \varphi_{\lambda_{j_{k}}}^{\mathbb{C}}(t+i \tau)\right|^{2} \leq 2(|\tau|-\epsilon) \text { on all of } \partial S_{\tau} .
$$

q.e.d.

7.1. Hartogs theorem for the family of translates. In this section, we prove a slightly more general version in which the origin $(x, \xi)$ is allowed to move with the index of the sequence:

Lemma 7.1. For any compact analytic Riemannian manifold $(M, g)$ of any dimension $m$, any complexified geodesic $\gamma_{x, \xi}$, and sequence $\left\{N_{j}\right\} \subset$ $\mathbb{R}$ and any sequence of eigenfunctions, the family of plurisubharmonic functions

$$
v_{j}:=\frac{1}{\pi \lambda_{j}} \log \left|\gamma_{G^{N_{j}(x, \xi)}}^{*} \varphi_{\lambda_{j}}^{\mathbb{C}}(t+i \tau)\right|^{2}
$$


is precompact in $L_{l o c}^{1}\left(S_{\epsilon}\right)$ as long as it does not converge uniformly to $-\infty$ on all compact subsets of $S_{\tau}$. Moreover, any limit of a subsequence is $\leq 2|\tau|$. If the limit $v$ of a subsequence $v_{j_{k}}$ is $\leq 2 \tau-\epsilon$ on an open interval $t \in(a, b)$, then $v \leq 2|\tau|-\epsilon$ for all $t \in \mathbb{R}$ and

$$
\limsup _{k \rightarrow \infty} v_{j_{k}} \leq 2|\tau|-\epsilon
$$

Proof. The uniform upper bound by $2|\tau|$ of course holds for the whole family $\mathcal{F}(11)$. We also use Lemma 6.5 in place of Proposition 2 and with $Y_{j_{k}}^{x, \xi, I}$ in place of $U_{j_{k}}^{x, \xi, I}$ where $I$ is any one of the intervals in the proof of Lemma 1, and then follow the same argument.

q.e.d.

\section{Periodic geodesics on surfaces: Proof of Proposition 1}

In this section we prove Proposition 1 and therefore Theorem 1 for restrictions to periodic geodesics on real analytic surfaces with ergodic geodesic flow. We thus assume that $\gamma_{x, \xi}$ is a periodic orbit of period $L$. We then denote the orbital Fourier coefficients of an eigenfunction by

$$
\nu_{j}^{x, \xi}(n)=\frac{1}{L_{\gamma}} \int_{0}^{L_{\gamma}} \varphi_{j}\left(\gamma_{x, \xi}(t)\right) e^{-\frac{2 \pi i n t}{L}} d t .
$$

Thus, we have

$$
\varphi_{j}\left(\gamma_{x, \xi}(t)\right)=\sum_{n \in \mathbb{Z}} \nu_{j}^{x, \xi}(n) e^{\frac{2 \pi i n t}{L}} .
$$

Hence the analytic continuation is given by

$$
\varphi_{j}^{\mathbb{C}}\left(\gamma_{x, \xi}(t+i \tau)\right)=\sum_{n \in \mathbb{Z}} \nu_{j}^{x, \xi}(n) e^{\frac{2 \pi i n(t+i \tau)}{L}} .
$$

It follows from the Paley-Wiener theorem and from the fact that $\gamma_{x, \xi}^{*} \varphi_{j}$ admits an analytic continuation to the annulus $e^{-|\tau|}<r<e^{|\tau|}$ that $\left|\nu_{j}^{x, \xi}(n)\right| \leq C_{j} e^{-|n| \tau}$. Furthermore, the Fourier modes $|n|>>\lambda_{j}$ are exponentially decaying. In semi-classical language, such high angular momentum is inconsistent with the energy $\lambda_{j}^{2}$ of the particle. More precisely, for $n \geq \lambda_{j}$,

$$
\left|\nu_{j}^{x, \xi}(n)\right|^{2} \leq \lambda_{j}^{\frac{m-1}{2}} e^{2|\tau|\left(\lambda_{j}-n\right)}
$$

Indeed, Proposition 3.9 gives

$$
\frac{1}{L_{\gamma}} \int_{\gamma_{x, \xi}^{\tau}}\left|\varphi_{j}^{\mathbb{C}}\right|^{2} d s \leq \lambda_{j}^{\frac{m-1}{4}} e^{2|\tau| \lambda_{j}}
$$

It follows that

$$
\sum_{n}\left|\nu_{j}^{x, \xi}(n)\right|^{2} e^{2 n|\tau|} \leq \lambda_{j}^{\frac{m-1}{4}} e^{2|\tau| \lambda_{j}}
$$


and (76) follows immediately from

$$
\sum_{n \geq \lambda_{j}}\left|\nu_{j}^{x, \xi}(n)\right|^{2} e^{2 n|\tau|} \leq \lambda_{j}^{\frac{m-1}{4}} e^{2|\tau| \lambda_{j}}
$$

8.1. Mass in the highest allowed modes. The purpose of this section is to prove

Proposition 8.1. Let $\operatorname{dim} M=2$. Suppose that $\left\{\varphi_{\lambda_{j}}\right\}$ is $Q E R$ along the periodic geodesic $\gamma_{x, \xi}$. Then for all $\epsilon>0$, there exists $C_{\epsilon}>0$ so that

$$
\sum_{n:|n| \geq(1-\epsilon) \lambda_{j}}\left|\nu_{\lambda_{j}}^{x, \xi}(n)\right|^{2} \geq C_{\epsilon} .
$$

Consequently,

$$
\sum_{n:|n| \geq(1-\epsilon) \lambda_{j}}\left|\nu_{\lambda_{j}}^{x, \xi}(n)\right|^{2} e^{-n \tau} \geq C_{\epsilon} e^{\tau(1-\epsilon) \lambda_{j}} .
$$

Proof. The first step is a direct application of the QER theorem of [TZ]. See Theorem 10.2. It implies that within the range $|n| \leq \lambda_{j}$ the Fourier coefficients are asymptotically all of the same size $\frac{1}{\lambda}$.

Lemma 8.2. Assume that $\gamma_{x, \xi}^{*} \varphi_{j}$ is QER. Then for any $0<a<b \leq$ 1 we have

$$
\sum_{n: a \lambda_{j}|n| \leq b \lambda_{j}}\left|\nu_{j}^{x, \xi}(n)\right|^{2} \rightarrow \int \chi_{[a, b]} d \mu_{x, \xi}
$$

Here, $d \mu_{x, \xi}$ denotes the limit measure in Theorem 10.2.

One can prove this using homogeneous or semi-classical pseudo-differential operators. For instance, let $\chi_{\lambda}(D)$ be a semi-classical convolution operator on the circle $S_{L}^{1}=\mathbb{R} / \frac{2 \pi}{L} \mathbb{Z}$ with $D=\frac{1}{i} \frac{d}{d t}$ and consider

$$
\left\langle\chi_{\lambda}(D) \gamma_{x, \xi}^{*} \varphi_{j}, \gamma_{x, \xi}^{*} \varphi_{j}\right\rangle_{S_{L}^{1}}=\sum_{n \in \mathbb{Z}} \chi\left(\frac{2 \pi i n}{L \lambda}\right)\left|\nu_{j}^{x, \xi}(n)\right|^{2} .
$$

Assuming $\left\{\varphi_{j}\right\}$ satisfies QER with respect to $\gamma_{x, \xi}$, we have

$$
\left\langle\chi_{\lambda}(D) \gamma_{x, \xi}^{*} \varphi_{j}, \gamma_{x, \xi}^{*} \varphi_{j}\right\rangle_{S_{L}^{1}} \rightarrow \int_{B^{*} S_{L}^{1}} \chi d \mu_{x, \xi}
$$

It follows that, for any $\epsilon>0$ we have

$$
\sum_{n:(1-\epsilon) \lambda_{j} \leq|n| \leq(1+\epsilon) \lambda_{j}}\left|\nu_{j}^{x, \xi}(n)\right|^{2} \rightarrow 2 \epsilon .
$$

Consequently,

$$
\sum_{n:|n| \geq(1-\epsilon) \lambda_{j}}\left|\nu_{\lambda_{j}}^{x, \xi}(n)\right|^{2} e^{-2 n \tau} \geq C_{\epsilon} e^{2 \tau(1-\epsilon) \lambda_{j}} .
$$


8.2. Completion of proof of Proposition 1 for periodic geodesics. The following Lemma is an integrated version of Proposition 1 .

Lemma 8.3. Let $\operatorname{dim} M=2$ and assume that $\left\{\varphi_{j}\right\}$ satsifies $Q E R$ along the periodic geodesic $\gamma$. Let $\left\|\gamma_{x, \xi}^{\tau *} \varphi_{j}^{\mathbb{C}}\right\|_{L^{2}\left(\partial S_{\tau}^{L}\right)}^{2}$ be the $L^{2}$-norm of the complexified restriction of $\varphi_{j}$ along one period cell $\partial S_{\tau}^{L}$. Then,

$$
\lim _{\lambda_{j} \rightarrow \infty} \frac{1}{\lambda_{j}} \log \left\|\gamma_{x, \xi}^{\tau *} \varphi_{j}^{\mathbb{C}}\right\|_{L^{2}\left(\partial S_{\tau}\right)}^{2} \rightarrow 2|\tau| .
$$

Indeed, this follows from Proposition 8.1 since, for any $\epsilon>0$,

$$
\liminf _{\lambda_{j} \rightarrow \infty} \log \sum_{n:|n| \geq(1-\epsilon) \lambda_{j}}\left|\nu_{\lambda_{j}}^{x, \xi}(n)\right|^{2} e^{-2 n \tau} \geq 2|\tau|(1-\epsilon) .
$$

To prove Proposition 1 we argue by contradiction. If it is false, then there exists a time interval $[a, b]$ and $\epsilon_{0}>0$ so that

$$
\int_{a}^{b}\left|\left(\gamma_{x, \xi}^{\tau}\right)^{*} \varphi_{j}^{\mathbb{C}}\right|^{2}=O\left(e^{\left(2|\tau|-\epsilon_{0}\right) \lambda_{j}}\right)
$$

On the other hand, by Proposition 8.1 we know that over the whole period interval we have,

$$
\int_{0}^{L}\left|\left(\gamma_{x, \xi}^{\tau}\right)^{*} \varphi_{j}^{\mathbb{C}}\right|^{2} \geq C\left(e^{(2|\tau|-\epsilon) \lambda_{j}}\right)
$$

for all $\epsilon>0$. Hence, we have

$$
\int_{a}^{b}\left|U_{j}^{x, \xi, \tau, T}\right|^{2} d t=O\left(e^{-\epsilon_{0} \lambda_{j}}\right) .
$$

But by Proposition 2, every weak* limit of $\left\{U_{j}^{x, \xi, \tau, T}\right\}$ is a constant multiple of Lebesgue measure. It follows that the multiple must be zero. But this contradicts Lemma 8.3.

\section{Non-periodic geodesics: Proof of Theorem 2}

In the periodic case, a key step is to compute the $L^{2}$ norm of the analytic continuation using the Plancherel theorem and to compare it to the $L^{2}$ norm in the real domain using the Plancherel theorem. To generalize the proof to non-periodic geodesics, we need to introduce a decaying analytic factor to put $\gamma_{x, \xi}^{*} \varphi_{j}^{\mathbb{C}}$ into $L^{2}$ along horizontal lines.

9.1. Analytic convergence factors. Let $\mathcal{G}$ be a real analytic function whose analytic extension to $S_{\tau}$ lies in $L^{2}\left(\partial S_{\tau}, d t\right)$ for each $\tau<\epsilon$. In particular we have in mind $\mathcal{G}(x)=e^{-x^{2} / 2}$, but a less rapidly decaying choice is $\mathcal{G}=\frac{1}{t+i p}$ for large enough $|p|>>\epsilon$. 
Thus for a given analytic and decaying convergence factor $\mathcal{G}$, we consider

$$
\left.\nu_{\lambda}^{x, \xi, \mathcal{G}}(\sigma)=\mathcal{F}\left(\mathcal{G} \cdot \gamma_{x, \xi}^{*} \varphi_{\lambda}\right)\right)(\sigma)=\int_{\mathbb{R}} \mathcal{G} \cdot \varphi_{\lambda}\left(\gamma_{x, \xi}(t)\right) e^{-i t \sigma} d t .
$$

We then have the Fourier inversion formula

$$
\left.\mathcal{G} \cdot \gamma_{x, \xi}^{*} \varphi_{\lambda_{j}}(s)=\int_{\mathbb{R}} e^{i s \sigma} \nu_{\lambda}^{x, \xi, \mathcal{G}}(\sigma)\right) d \sigma
$$

and analytically continue $\mathcal{G} \cdot \gamma_{x, \xi}^{*} \varphi_{\lambda_{j}}$ to

$$
\mathcal{G} \cdot \gamma_{x, \xi}^{*} \varphi_{\lambda_{j}}^{\mathbb{C}}(s+i \tau)=\int_{\mathbb{R}} e^{i(s+i \tau) \sigma} \nu_{\lambda}^{x, \xi, \mathcal{G}}(\sigma) d \sigma .
$$

We then have the Plancherel theorem for each fixed $\tau$

$$
\int_{-\infty}^{\infty} \mid \mathcal{G}\left(\left.\gamma_{x, \xi}^{*} \varphi_{\lambda_{j}}^{\mathbb{C}}(s+i \tau)\right|^{2} d s=\int_{\mathbb{R}} e^{-2|\tau| \sigma}\left|\nu_{\lambda_{j}}^{x, \xi, \mathcal{G}}(\sigma)\right|^{2} d \sigma .\right.
$$

As in the periodic case, the growth rate of $\left|\gamma_{x, \xi}^{*} \varphi_{\lambda_{j}}^{\mathbb{C}}(s+i \tau)\right|$ as $\lambda_{j} \rightarrow \infty$ depends on the magnitude of the Fourier transform $\nu_{\lambda_{j}}^{x, \xi}(\sigma)$ for $|\sigma| \simeq \lambda_{j}$.

Lemma 9.1. Let $\operatorname{dim} M=2$ and suppose that $\gamma_{x, \xi}$ is a non-periodic geodesic such that $Q E R$ holds in the real domain along each finite arc (such as a uniform geodesic.) Then for all $\epsilon>0$, there exists $C_{\epsilon}>0$ so that

$$
\int_{|\sigma| \geq(1-\epsilon) \lambda_{j}}\left|\nu_{\lambda_{j}}^{x, \xi, \mathcal{G}}(\sigma)\right|^{2} d \sigma \geq C_{\epsilon}
$$

Consequently,

$$
\int_{|\sigma| \geq(1-\epsilon) \lambda_{j}}\left|\nu_{\lambda_{j}}^{x, \xi, \mathcal{G}}(\sigma)\right|^{2} e^{-2 \sigma \tau} d \sigma \geq C_{\epsilon} e^{2|\tau|(1-\epsilon) \lambda_{j}} .
$$

Proof. We consider the test operator $\overline{\mathcal{G}} \chi\left(\lambda^{-1} D\right) \mathcal{G}$ in the real domain, and its matrix elements

$$
\left.\left\langle\chi\left(\lambda_{j}^{-1} D\right) \mathcal{G} \gamma_{x, \xi}^{*} \varphi_{j}, \mathcal{G} \gamma_{x, \xi}^{*} \varphi_{j}\right\rangle_{L^{2}(\mathbb{R})}=\left\langle\overline{\mathcal{G}} \chi\left(\lambda_{j}^{-1} D\right) \mathcal{G} \gamma_{x, \xi}^{*} \varphi_{j}, \gamma_{x, \xi}^{*} \varphi_{j}\right\rangle\right\rangle_{L^{2}(\mathbb{R})} .
$$

Claim 9.2. There exists a subsequence of eigenvalues $\left\{\lambda_{j_{k}}\right\}$ of density one so that,

$$
\lim _{k \rightarrow \infty}\left\langle\chi\left(\lambda_{j_{k}}^{-1} D\right) \mathcal{G} \gamma_{x, \xi}^{*} \varphi_{j_{k}}, \mathcal{G} \gamma_{x, \xi}^{*} \varphi_{j_{k}}\right\rangle=\int_{B^{*} \mathbb{R}}|\mathcal{G}(t)|^{2} \chi(\sigma) d t d \sigma
$$

This is almost the QER Theorem 10.2 except that the operator $\chi\left(\lambda_{j}^{-1} D\right) \mathcal{G}$ does not have compact spatial support in the $t$ variable. To deal this, we introduce a cutoff in $t$ and then verify that it can be removed. 
Theorem 10.2 implies that

$$
\lim _{k \rightarrow \infty}\left\langle\mathbf{1}_{[-T, T]} \chi\left(\lambda_{j_{k}}^{-1} D\right) \mathcal{G} \gamma_{x, \xi}^{*} \varphi_{j_{k}}, \mathcal{G} \gamma_{x, \xi}^{*} \varphi_{j_{k}}\right\rangle=\int_{B^{*} \mathbb{R}}\left\langle\mathbf{1}_{[-T, T]}|\mathcal{G}(t)|^{2} \chi(\sigma) d t d \sigma\right.
$$

On the other hand, we claim that there exists a subsequence of $\left\{\lambda_{j_{k}}\right\}$ of natural density one so that the $\mathcal{G}$-weighted mass on the complement of $[T, T]$ is arbitrarily small, i.e. for all $\epsilon$ there exists $T(\epsilon)$ so that for $T \geq T(\epsilon)$,

$$
\limsup _{k \rightarrow \infty}\left|\left\langle\mathbf{1}_{|t| \geq T} \chi\left(\lambda_{j_{k}}^{-1} D\right) \mathcal{G} \gamma_{x, \xi}^{*} \varphi_{j_{k}}, \mathcal{G} \gamma_{x, \xi}^{*} \varphi_{j_{k}}\right\rangle\right| \leq \epsilon .
$$

To prove this, we consider the Weyl sums

$$
N(\lambda, T, \mathcal{G})=\sum_{j: \lambda_{j} \leq \lambda}\left|\left\langle\mathbf{1}_{|t| \geq T} \chi\left(\lambda_{j_{k}}^{-1} D\right) \mathcal{G} \gamma_{x, \xi}^{*} \varphi_{j_{k}}, \mathcal{G} \gamma_{x, \xi}^{*} \varphi_{j_{k}}\right\rangle\right| .
$$

Assuming with no loss of generality that we use a positive quantization, this sum is bounded above by

$$
\begin{aligned}
N(\lambda, T, \mathcal{G}) & \leq \sum_{j: \lambda_{j} \leq \lambda}\left\langle\mathbf{1}_{|t| \geq T}\left|\overline{\mathcal{G}} \chi\left(\lambda_{j_{k}}^{-1} D\right) \mathcal{G}\right| \gamma_{x, \xi}^{*} \varphi_{j_{k}}, \gamma_{x, \xi}^{*} \varphi_{j_{k}}\right\rangle \\
& =\int_{T^{*} \mathbb{R}} \mathbf{1}_{|t| \geq T} \sigma\left(|\overline{\mathcal{G}}| \chi\left(\lambda_{j_{k}}^{-1} D\right) \mathcal{G} \mid\right) \sum_{j: \lambda_{j} \leq \lambda} d W_{j}^{x, \xi}
\end{aligned}
$$

Then

$$
\lim _{\lambda \rightarrow \infty} \frac{N(\lambda, T, \mathcal{G})}{N(\lambda)} \leq \int_{B^{*} \mathbb{R}} \mathbf{1}_{|t| \geq T}|\mathcal{G}|^{2} \chi(\sigma) d s d \sigma<\epsilon,
$$

if $T$ is chosen large enough so that $\int_{|t| \geq T}|\mathcal{G}|^{2} d s<\epsilon$. By definition, a density one proportion terms of the series in the numerator must be $<2 \epsilon$.

On the other hand we can use (79) with $\tau=0$ to get

$$
\int_{\mathbb{R}} \chi\left(\sigma / \lambda_{j}\right)\left|\nu_{\lambda_{j}}^{x, \xi, \mathcal{G}}(\sigma)\right|^{2} d t \simeq \int_{|\sigma| \geq(1-\epsilon) \lambda_{j}}\left|\nu_{\lambda_{j}}^{x, \xi, \mathcal{G}}(\sigma)\right|^{2} d t .
$$

Combining the two limit formulae gives, for any $\epsilon>0$,

$$
\liminf _{\lambda_{j} \rightarrow \infty} \int_{|\sigma| \geq(1-\epsilon) \lambda_{j}}\left|\nu_{\lambda_{j}}^{x, \xi, \mathcal{G}}(\sigma)\right|^{2} d t \geq 2 \epsilon\|\mathcal{G}\|_{L^{2}(\mathbb{R})}^{2} .
$$

In the complex domain on $\partial S_{\tau}$ we then use (80) to get

$$
\liminf _{\lambda_{j} \rightarrow \infty} \int_{|\sigma| \geq(1-\epsilon) \lambda_{j}}\left|\nu_{\lambda_{j}}^{x, \xi, \mathcal{G}}(\sigma)\right|^{2} e^{-2 \sigma \tau} d \sigma \geq 2 \epsilon|| \mathcal{G} \|_{L^{2}(\mathbb{R})}^{2} \cdot e^{2|\tau|(1-\epsilon) \lambda_{j}},
$$

concluding the proof of the Lemma. 
9.2. Logarithmic asymptotics. As in the periodic case, we can then compute logarithmic asymptotics of $L^{2}$ norms on $\partial S_{\tau}$ :

Lemma 9.3. Assume that $\left\{\varphi_{j}\right\}$ satisfies QER along arcs of the geodesic $\gamma_{x, \xi}$. Let $\left\|\mathcal{G} \gamma_{x, \xi}^{\tau *} \varphi_{j}^{\mathbb{C}}\right\|_{L^{2}\left(\partial S_{\tau}\right)}$ be the $L^{2}$-norm along $\partial S_{\tau}$. Then for all $\mathcal{G}$ as above,

$$
\lim _{\lambda_{j} \rightarrow \infty} \frac{1}{\lambda_{j}} \log \left\|\mathcal{G} \gamma_{x, \xi}^{\tau *} \varphi_{j}^{\mathbb{C}}\right\|_{L^{2}\left(\partial S_{\tau}\right)}^{2}=2|\tau| .
$$

Proof. By Lemma 9.1, we have for any $\epsilon>0$, and any $\mathcal{G}$ as above,

$$
\begin{aligned}
& \liminf _{\lambda_{j} \rightarrow \infty} \frac{1}{\lambda_{j}} \log \left\|\mathcal{G} \gamma_{x, \xi}^{* \tau} \varphi_{j}^{\mathbb{C}}\right\|_{L^{2}\left(\partial S_{\tau}\right)}^{2} \\
= & \liminf _{\lambda_{j} \rightarrow \infty} \frac{1}{\lambda_{j}} \log \int_{\mathbb{R}} e^{-2 \tau \sigma}\left|\nu_{\lambda_{j}}^{x, \xi, \mathcal{G}}(\sigma)\right|^{2} d \sigma . \\
\geq & 2(1-\epsilon)|\tau|
\end{aligned}
$$

On the other hand, the upper bound follows (as usual) by Proposition 3.9 .

q.e.d.

Let $\mathcal{G}(t)=\frac{1}{t+i p}$ where $|p|$ is sufficiently large (as above). Lemma 9.1 implies:

Corollary 9.4. For any $\epsilon>0$ and any $\lambda_{j}$ there exists $N_{j}=N\left(\epsilon, \lambda_{j}\right) \epsilon$ $\mathbb{R}$ so that

$$
\liminf _{\lambda_{j} \geq \infty} \frac{1}{\lambda_{j}} \log \int_{N_{j}}^{N_{j}+1}\left|\gamma_{x, \xi}^{* \tau} \varphi_{j}^{\mathbb{C}}\right|^{2} d t>2|\tau|-\epsilon .
$$

Here, the choice of $\left[N_{j}, N_{j}+1\right]$ is somewhat arbitrary; there is no need to make the intervals of unit length.

Proof. Using obvious upper bounds on $\mathcal{G}$ on the intervals $[n, n+1]$ we have,

$$
\int_{-\infty}^{\infty} \mid \mathcal{G}\left(\left.\gamma_{x, \xi}^{*} \varphi_{\lambda_{j}}^{\mathbb{C}}(s+i \tau)\right|^{2} d s \leq \sum_{n \in \mathbb{Z}} \frac{1}{1+n^{2}} \int_{n+1}^{n}\left|\gamma_{x, \xi}^{* \tau} \varphi_{j}^{\mathbb{C}}\right|^{2} d t .\right.
$$

Hence by Lemma 9.1 ,

$$
2|\tau| \leq \liminf _{\lambda_{j} \geq \infty} \frac{1}{\lambda_{j}} \log \sum_{n \in \mathbb{Z}} \frac{1}{1+n^{2}} \int_{n+1}^{n}\left|\gamma_{x, \xi}^{* \tau} \varphi_{j}^{\mathbb{C}}\right|^{2} d t .
$$

If the Lemma were false, we would have for all $n$, and sufficienty large $\lambda_{j}$,

$$
\int_{n}^{n}\left|\gamma_{x, \xi}^{* \tau} \varphi_{j}^{\mathbb{C}}\right|^{2} d t<e^{2|\tau|-\epsilon}
$$

and then $\frac{1}{\lambda_{j}} \log \sum_{n \in \mathbb{Z}} \frac{1}{1+n^{2}}$ of these integrals would be $\leq 2|\tau|-\epsilon$, contradicting the lower bound (83). 
9.3. Completion of proof of Theorem 2. As in the previous cases, we need to rule out (73).

By Lemma (9.3) and Corollary 9.4 there exists a sequence $\left[N_{j}, N_{j+1}\right]$ for which the lower bound of Corollary 9.4 holds. For this sequence, (9) is false. It follows by Lemma 7.1 that $v_{j} \rightarrow 2|\tau|$. The rest of the proof of Theorem 2 follows as in the periodic case.

\section{Appendix}

In this appendix, we review the QER result of $[\mathbf{T Z}]$ (see also $[\mathbf{D Z}]$ ). We also review the theory of Fourier integral operators with complex phase that we use in this article. We refer to $[\mathbf{M S j}]$ and volume IV of $[\mathbf{H o}]$ for background. Since the manifolds and metrics in this article are real analytic, the theory of almost analytic extensions is not needed.

10.1. Quantum ergodic restriction in the real domain. In this section we review the QER theorem for hypersurfaces of $[\mathbf{T Z}]$. There is no advantage to specializing to curves in surfaces, so we review the result for hypersurfaces $H \subset M$.

Let $H \subset M$ be an embedded submanifold, and denote by

$$
T_{H}^{*} M=\left\{(q, \xi) \in T_{q}^{*} M, \quad q \in H\right\}
$$

the cotangent bundle of the ambient space along $H$. We also denote by $T^{*} H=\left\{(q, \eta) \in T_{q}^{*} H, \quad q \in H\right\}$ the cotangent bundle of $H$. We further denote by $r_{H}: T_{H}^{*} M \rightarrow T_{H}^{*} M$ the reflection map through $T^{*} H$, i.e. $r_{H}(\xi)=\xi^{\prime}$ with $\left.\xi\right|_{T H}=\left.\xi^{\prime}\right|_{T H}$ but with opposite normal components.

We define the first return time $T(s, \xi)$ on $S_{H}^{*} M$ by,

$$
\left.T(s, \xi)=\inf \left\{t>0: G^{t}(s, \xi) \in S_{H}^{*} M, \quad(s, \xi) \in S_{H}^{*} M\right)\right\} .
$$

By definition $T(s, \xi)=+\infty$ if the trajectory through $(s, \xi)$ fails to return to $H$. We define the first return map on the same domain by

$$
\Phi: S_{H}^{*} M \rightarrow S_{H}^{*} M, \quad \Phi(s, \xi)=G^{T(s, \xi)}(s, \xi)
$$

When $G^{t}$ is ergodic, $\Phi$ is defined almost everywhere and is also ergodic.

Definition 4. We say that $H$ has a positive measure of microlocal reflection symmetry if

$\mu_{L, H}\left(\bigcup_{j \neq 0}^{\infty}\left\{(s, \xi) \in S_{H}^{*} M: r_{H} G^{T^{(j)}(s, \xi)}(s, \xi)=G^{T^{(j)}(s, \xi)} r_{H}(s, \xi)\right\}\right)>0$.

Otherwise we say that $H$ is asymmetric with respect to the geodesic flow.

To state the QER result for Dirichlet data, we need some further notation. The result holds for several classes of pseudo-differential operators on $H$ with essentially the same proof. We state the result first for 
pseudo-differential operators with classical poly-homogeneous symbols

$$
a(s, \sigma) \sim \sum_{k=0}^{\infty} a_{-k}(s, \sigma),\left(a_{-k} \text { positive homogeneous of order }-k\right)
$$

on $T^{*} H$ and then for semi-classical pseudo-differential operators with semi-classical symbols $a \in S^{0,0}\left(T^{*} H \times\left(0, h_{0}\right]\right.$ of the form

$$
a_{\hbar}(s, \sigma) \sim \sum_{k=0}^{\infty} \hbar^{k} a_{-k}(s, \sigma),\left(a_{-k} \in S_{1,0}^{-k}\left(T^{*} H\right)\right)
$$

as in $[\mathbf{H Z}, \mathbf{T Z}]$.

The restriction map $S_{H}^{*} M \rightarrow B^{*} H$ is singular along $S^{*} H$ and pushes forward Liouville measure to a multiple $\gamma_{B^{*} H}^{-1} d s d \sigma$ of the symplectic volume density on $B^{*} H$. Here, $\gamma_{B^{*} H}:=\left(1-|\sigma|^{2}\right)^{\frac{1}{2}}$ We note that $\gamma$ is a zeroth-order homogeneous function on $T_{H}^{*} M$ which equals the nonhomogeneous $\gamma_{B^{*} H}$ of $[\mathbf{H Z}]$ on $S_{H}^{*} M$ (i.e. $\left|\eta_{n}\right|^{2}+|\sigma|^{2}=1$ ).

For homogeneous pseudo-differential operators, the QER theorem is as follows:

Theorem 10.1. Let $(M, g)$ be a compact manifold with ergodic geodesic flow, and let $H \subset M$ be a hypersurface. Let $\varphi_{\lambda_{j}} ; j=1,2, \ldots$ denote the $L^{2}$-normalized eigenfunctions of $\Delta_{g}$. If $H$ has a zero measure of microlocal symmetry, then there exists a density-one subset $S$ of $\mathbb{N}$ such that for $\lambda_{0}>0$ and $a(s, \sigma) \in S_{c l}^{0}\left(T^{*} H\right)$

$$
\lim _{\lambda_{j} \rightarrow \infty ; j \in S}\left\langle\left. O p(a) \varphi_{\lambda_{j}}\right|_{H},\left.\varphi_{\lambda_{j}}\right|_{H}\right\rangle_{L^{2}(H)}=\omega(a),
$$

where

$$
\omega(a)=\frac{4}{\operatorname{vol}\left(S^{*} M\right)} \int_{B^{*} H} a_{0}(s, \sigma) \gamma_{B^{*} H}^{-1}(s, \sigma) d s d \sigma .
$$

The analogous result for semi-classical pseudo-differential operators is:

Theorem 10.2. Let $(M, g)$ be a compact manifold with ergodic geodesic flow, and let $H \subset M$ be a hypersurface. If $H$ has a zero measure of microlocal symmetry, then there exists a density-one subset $S$ of $\mathbb{N}$ such that for $a \in S^{0,0}\left(T^{*} H \times\left[0, h_{0}\right)\right)$,

$$
\lim _{h_{j} \rightarrow 0^{+} ; j \in S}\left\langle\left. O p_{h_{j}}(a) \varphi_{h_{j}}\right|_{H},\left.\varphi_{h_{j}}\right|_{H}\right\rangle_{L^{2}(H)}=\omega(a),
$$

where

$$
\omega(a)=\frac{4}{\operatorname{vol}\left(S^{*} M\right)} \int_{B^{*} H} a_{0}(s, \sigma) \gamma_{B^{*} H}^{-1}(s, \sigma) d s d \sigma .
$$


10.2. Fourier integral distributions with complex phase. First, we review the relevant definitions (see $[\mathbf{H o}] \mathrm{IV}, \S 25.5$ or $[\mathbf{M S j}]$ ). A Fourier integral distribution with complex phase on a manifold $X$ is a distribution that can locally be represented by an oscillatory integral

$$
A(x)=\int_{\mathbb{R}^{N}} e^{i \varphi(x, \theta)} a(x, \theta) d \theta
$$

where $a(x, \theta) \in S^{m}(X \times V)$ is a symbol of order $m$ in a cone $V \subset \mathbb{R}^{N}$ and where the phase $\varphi$ is a positive regular phase function, i.e. it satisfies

- $\Im \varphi \geq 0$

- $d \frac{\partial \varphi}{\partial \theta_{1}}, \ldots, d \frac{\partial \varphi}{\partial \theta_{N}}$ are linearly independent complex vectors on

$$
C_{\varphi \mathbb{R}}=\left\{(x, \theta): d_{\theta}(x, \theta)=0\right\} .
$$

- In the analytic setting (which is assumed in this article), $\varphi$ admits an analytic continuation $\varphi_{\mathbb{C}}$ to an open cone in $X_{\mathbb{C}} \times V_{\mathbb{C}}$.

Define

$$
C_{\varphi_{\mathbb{C}}}=\left\{(x, \theta) \in X_{\mathbb{C}} \times V_{\mathbb{C}}: \nabla_{\theta} \varphi_{\mathbb{C}}(x, \theta)=0\right\} .
$$

Then $C_{\varphi_{\mathbb{C}}}$ is a manifold near the real domain. One defines the Lagrangian submanifold $\Lambda_{\varphi_{\mathbb{C}}} \subset T^{*} X_{\mathbb{C}}$ as the image

$$
(x, \theta) \in C_{\varphi_{\mathbb{C}}} \rightarrow\left(x, \nabla_{x} \varphi_{\mathbb{C}}(x, \theta)\right) .
$$

According to Definition 4.4 of $[\mathbf{M S j}]$, the space $I^{m}(X, \Lambda)$ of Fourier integral operators of order $m$ with complex phase is the class of operators satisfying

- $W F(A) \subset \Lambda_{\mathbb{R}}$

- For every $\lambda_{0} \in \Lambda_{\mathbb{R}}$ and every choice of local coordinates $x_{1}, \ldots, x_{n}$ near $\pi\left(\lambda_{0}\right), A$ is microlocally of the form $I(a, \varphi)$ near $\lambda_{0}$ where $\varphi$ is a positive phase function generating $\Lambda$ near $\lambda_{0}$ and where $a \in S^{m+(n+2 N) / 4}\left(\mathbb{R}^{n} \times \mathbb{R}^{N}\right)$ has its support in a small conic neighborhood of $\left(x_{0}, \theta_{0}\right) \in C_{\varphi \mathbb{R}}$, i.e. the point corresponding to $\lambda_{0}$.

Given a closed conic positive Lagrangian manifold $\Lambda \subset T^{*} \tilde{X}-0$, there exists a principal symbol map

$$
A \in I_{c}^{n}(X, \Lambda) / I_{c}^{m-1}(X, \Lambda) \rightarrow \Gamma^{m+n / 4}(\Lambda ; \mathcal{L}),
$$

and also a quantization (denoted $\mathcal{P}$ in $[\mathbf{M S j}]$ ) which inverts it. As in the real domain, given a real analytic phase $\varphi$ and its holomorphic extension $\tilde{\varphi}$ parametrizing $\Lambda$, one defines the Leray residue form $d_{\tilde{\varphi}}$ on $C_{\tilde{\varphi}}$ by

$$
d_{\tilde{\varphi}} \wedge d \frac{\partial \tilde{\varphi}}{\partial \tilde{\theta}_{1}} \wedge \cdots \wedge d \frac{\partial \tilde{\varphi}}{\partial \tilde{\theta}_{n}}=i^{n+N} d z_{1}, \cdots \wedge d z_{n} \wedge d \tilde{\theta}_{1} \wedge \cdots \wedge d \tilde{\theta}_{N} .
$$

If $I(\varphi, A)$ is a complex oscillatory integral with positive phase, and $a_{0}$ is the principal term of the amplitude $A$, then the symbol of $I(\varphi, A)$ is

$$
a_{0} \sqrt{d_{\tilde{\varphi}}} .
$$


10.3. Complex canonical relation of $\Pi_{\tau}$. We denote by $\widetilde{\partial M}_{\tau}$ the complexification of $\partial M_{\tau}$. The positive complex canonical relation of $\Pi_{\tau}$ is the idempotent canonical relation

$$
C_{\tau} \subset T^{*}\left(\widetilde{\partial M}_{\tau} \times \widetilde{\partial M}_{\tau}\right)
$$

satisfying $C_{\tau}^{2}=C_{\tau}=C_{\tau}^{*}$ generated by the complex extension $\rho(z, w)$ of the Grauert tube function to $M_{\tau} \times \overline{M_{\tau}}$. Thus,

$$
C_{\tau}=\left\{\left(z, \theta \partial_{z} \rho, w, \theta \bar{\partial}_{w} \rho\right): z \in \widetilde{\partial M}_{\tau}, w \in{\overline{\partial M_{\tau}}}, \theta \in \mathbb{R}_{+}, \rho(z, w)=4 \tau^{2} \cdot\right\} .
$$

The canonical relation $C_{\tau}$ can also be described as a flowout relation in terms of complex characteristics of the tangential Cauchy-Riemann operator $\bar{\partial}_{b}$. As a strongly pseudo-convex hypersurface in the complex manifold $M_{\mathbb{C}}, \partial M_{\tau}$ is a CR manifold whose complexified tangent bundle has a complex codimension one subspace invariant under the complex structure $J$. We denote by $Z_{1}, \ldots, Z_{m}$, resp. $\bar{Z}_{1}, \ldots, \bar{Z}_{m}$ an orthonormal basis with respect to the Kähler form $\omega_{\rho}$ on $M_{\mathbb{C}}$ of the holomorphic tangent space $T^{1,0} \partial M_{\tau}$, resp. the anti-holomorphic tangent space $T^{0,1}\left(\partial M_{\tau}\right)$. Then $\square_{b}=\sum_{j} \bar{Z}_{j}^{*} \bar{Z}_{j}$.

We denote the symbol of $\square_{b}$ by $q$. Its zero set is the characteristic variety $\Sigma_{\tau}$ of $\square_{b}$ in the real cotangent space $T^{*} \partial M_{\tau}$, i.e. simultaneous kernel of the functions

$$
\zeta_{j}(x, \xi)=\left\langle\xi, \bar{Z}_{j}\right\rangle
$$

which are the symbols of the associated derivative along $\bar{Z}_{j}$. Thus,

$$
q=\sum_{j=1}^{d}\left|\zeta_{j}\right|^{2}: T^{*} \partial M_{\tau} \rightarrow \mathbb{R} .
$$

When we holomorphically extend to $\widetilde{\partial M}_{\tau}$, we get the complex characteristic variety $\mathcal{J}_{+} \subset T^{*}\left(\widetilde{\partial M}_{\tau}\right)$, the zero set of $\tilde{q}$, the holomorphic extension of $q$. We let $\tilde{\zeta}_{j}$ be the analytic extensions to $\widetilde{\partial M}_{\tau}$ of the functions $\zeta_{j}$ and $\tilde{\sigma}$ be the standard holomorphic symplectic form of $T^{*} \widetilde{\partial M}_{\tau}$. Thus,

$$
\mathcal{J}_{+}=\left\{(\tilde{x}, \tilde{\xi}) \in T^{*} \widetilde{\partial M}_{\tau}: \tilde{\zeta}_{j}=0 \forall j\right\}=\{\tilde{q}=0\} .
$$

It is an involutive sub-manifold of $T^{*} \widetilde{\partial M}_{\tau}$ with the properties:

$$
\begin{array}{ll}
\text { (i) } & \left(\mathcal{J}_{+}\right)_{\mathbb{R}}=\Sigma \\
\text { (ii) } & \frac{1}{i} \tilde{\sigma}(u, \bar{u})>0, \forall u \in T\left(\mathcal{J}_{+}\right)^{\perp} \\
\text { (iii) } & T_{\rho}\left(\mathcal{J}_{+}\right)=T_{\rho} \tilde{\Sigma} \oplus W_{\rho}^{+} .
\end{array}
$$

Here, $W_{\rho}^{+}$is the sum of the eigenspaces of $F_{\rho}$, the normal Hessian of $q$, corresponding to the eigenvalues $\left\{i \lambda_{j}\right\}$ with $\lambda_{j} \geq 0$. 
Since $\mathcal{J}_{+}$is a co-isotropic (i.e. involutive) submanifold of $T^{*} \widetilde{\partial M}_{\tau}$, it has a null folation, which is given by the joint Hamilton flow of the defining functions $\tilde{\zeta}_{j}$. We then have,

$$
C_{\tau}:=\left\{(\tilde{x}, \tilde{\xi}, \tilde{y}, \tilde{\eta}) \in \mathcal{J}_{+} \times \overline{\mathcal{J}_{+}}:(\tilde{x}, \tilde{\xi}) \sim(\tilde{y}, \tilde{\eta})\right\}
$$

where $\sim$ is the equivalence relation of 'belonging to the same leaf of the null foliation of $\mathcal{J}_{+}$.

\section{References}

[BR] J. Bourgain \& Z. Rudnick, Restriction of toral eigenfunctions to hypersurfaces and nodal sets, Geom. Funct. Anal. 22 (2012), no. 4, 878-937. MR 2984120, Zbl pre06110797.

[Bou] L. Boutet de Monvel, Convergence dans le domaine complexe des séries de fonctions propres, C. R. Acad. Sci. Paris Sr. A-B 287 (1978), no. 13, A855A856. MR 0544506, Zbl 0392.35043.

[BoGu] L. Boutet de Monvel \& V. Guillemin, The Spectral Theory of Toeplitz Operators, Ann. Math. Studies 99, Princeton Univ. Press, Princeton, 1981. MR 0620794, Zbl 0469.47021.

[BoSj] L. Boutet de Monvel \& J. Sjöstrand, Sur la singularité des noyaux de Bergman et de Szegö, Asterisque 34-35 (1976), 123-164. MR 0590106, Zbl 0344.32010.

[BW] F. Bruhat \& H. Whitney, Quelques propriétés fondamentales des ensembles analytiques-réels, Comment. Math. Helv. 33 (1959) 132-160. MR 0102094, Zbl 0100.08101.

[DZ] S. Dyatlov \& M. Zworski, Quantum ergodicity for restrictions to hypersurfaces, Nonlinearity 26 (2013), no. 1, 35-52. MR 3001760.

[GRS] A. Ghosh, A. Reznikov, \& P. Sarnak, Nodal domains of Maass forms I, arXiv: 1207.6625 .

[GLS] F. Golse, E. Leichtnam, \& M. Stenzel, Intrinsic microlocal analysis and inversion formulae for the heat equation on compact real-analytic Riemannian manifolds, Ann. Sci. École Norm. Sup. (4) 29 (1996), no. 6, 669-736. MR 1422988, Zbl 0889.32037.

[GS1] V. Guillemin \& M. Stenzel, Grauert tubes and the homogeneous MongeAmpère equation, J. Differential Geom. 34 (1991), no. 2, 561-570. MR 1131444, Zbl 0746.32005.

[GS2] V. Guillemin \& M. Stenzel, Grauert tubes and the homogeneous MongeAmpère equation II, J. Differential Geom. 35 (1992), no. 3, 627-641. MR 1163451, Zbl 0789.32010.

[HZ] A. Hassell \& S. Zelditch, Quantum ergodicity of boundary values of eigenfunctions, Comm. Math. Phys. 248 (2004), no. 1, 119-168. MR 2104608, Zbl 1054.58022.

[Ho] L. Hörmander, The Analysis of Linear Partial Differential Operators, Grund. Math. Wiss. 256, 274 Springer-Verlag, New York, 1983. MR 2512677, Zbl 1178.35003 .

[Ho2] L. Hörmander, Notions of convexity. Reprint of the 1994 edition. Modern Birkhäuser Classics. Birkhäuser Boston, Inc., Boston, MA, 2007. MR 2311920, Zbl 1108.32001. 
ERGODICITY \& INTERSECTIONS OF NODAL SETS AND GEODESICS 351

[LS] L. Lempert \& R. Szöke, Global solutions of the homogeneous complex MongeAmpère equation and complex structures on the tangent bundle of Riemannian manifolds, Math. Ann. 290 (1991), no. 4, 689-712. MR 1119947, Zbl 0752.32008

[MSj] A. Melin \& J. Sjöstrand, Fourier integral operators with complex-valued phase functions. Fourier integral operators and partial differential equations (Colloq. Internat., Univ. Nice, Nice, 1974), pp. 120-223. Lecture Notes in Math., Vol. 459, Springer, Berlin, 1975. MR 0431289, Zbl 0306.42007.

[Shn] A. I. Shnirelman, Ergodic properties of eigenfunctions, Usp. Mat. Nauk. 29/6 (1974), 181-182. MR 0402834.

[St] M. Stenzel, (in preparation 2012).

[TZ] J. A. Toth \& S. Zelditch, Quantum ergodic restriction theorems, II: manifolds without boundary, Geom. Funct. Anal. 23 (2013), 715-775. MR 3053760.

[Z] S. Zelditch, Complex zeros of real ergodic eigenfunctions, Invent. Math. 167 (2007), no. 2, 419-443. MR 2270460, Zbl 1134.37005.

[Z2] S. Zelditch, Pluri-potential theory on Grauert tubes of real analytic Riemannian manifolds I, Spectral Geometry, Proc. Sympos. Pure Math. 84, Amer. Math. Soc., Providence, RI (2012), 299-339. MR 2985323.

[Z3] S. Zelditch, Index and dynamics of quantized contact transformations, Ann. Inst. Fourier (Grenoble) 47 (1997), no. 1, 305-363. MR 1437187, Zbl 0865.47018.

[Z4] S. Zelditch, Eigenfunctions and Nodal sets, Surveys in Differential Geometry volume 18 (2013), pp. 237-308.

[Zw] M. Zworski, Semiclassical analysis. Graduate Studies in Mathematics, 138. American Mathematical Society, Providence, RI (2012). MR 2952218.

\section{DePARTMENT OF MATHEMATiCs NORTHWESTERN UNIVERSITY EVANSTON, IL 60208, USA}

E-mail address: zelditch@math.northwestern.edu 